\title{
INFINITE PATHS AND CLIQUES IN RANDOM GRAPHS
}

\author{
ALESSANDRO BERARDUCCI, PIETRO MAJER AND MATTEO NOVAGA
}

\begin{abstract}
We study the thresholds for the emergence of various properties in random subgraphs of $(\mathbb{N},<)$. In particular, we give sharp sufficient conditions for the existence of (finite or infinite) cliques and paths in a random subgraph. No specific assumption on the probability is made. The main tools are a topological version of Ramsey theory, exchangeability theory and elementary ergodic theory.
\end{abstract}

\section{Contents}

1. Introduction

2. Notations

3. Finite paths in random subgraphs

4. Infinite paths

5. Threshold functions for graph morphisms

5.1. Chromatic number

6. Infinite cliques

Appendix A. A topological Ramsey theorem

Appendix B. Exchangeable measures

References

\section{INTRODUCTION}

In this paper we introduce a new method in order to deal with some combinatorial problems in random graphs, originally proposed in EH:64. Some of this questions have been successfully addressed in [FT:85, using different techniques. We obtain new and self-contained proofs of some of the results in FT:85]; moreover with this method we expect to be able to treat similar problems in more general random graphs.

Let $G=\left(\mathbb{N}, \mathbb{N}^{(2)}\right)$ be the directed graph over $\mathbb{N}$ with set of edges $\mathbb{N}^{(2)}:=$ $\left\{(i, j) \in \mathbb{N}^{2}: i<j\right\}$. Let us randomly choose some of the edges of $G$, that is, we associate to the edge $(i, j) \in \mathbb{N}^{(2)}$ a measurable set $\mathbb{X}_{i, j} \subseteq \Omega$, where $(\Omega, \mathcal{A}, \mu)$ is a base probability space. Assuming $\mu\left(\mathbb{X}_{i, j}\right) \geq \lambda$ for each $(i, j)$, we then ask whether the resulting random subgraph $\mathbb{X}$ of $\left(\mathbb{N}, \mathbb{N}^{(2)}\right)$ contains an infinite path:

Problem 1. Let $(\Omega, \mathcal{A}, \mu)$ be a probability space. Let $\lambda>0$ and for all $(i, j) \in \mathbb{N}^{(2)}$, let $\mathbb{X}_{i, j}$ be a measurable subset of $\Omega$ with $\mu\left(\mathbb{X}_{i, j}\right) \geq \lambda$. Is there an infinite increasing sequence $\left\{n_{i}\right\}_{i \in \mathbb{N}}$ such that $\bigcap_{i \in \mathbb{N}} \mathbb{X}_{n_{i}, n_{i+1}}$ is non-empty? 
More formally, a random subgraph $\mathbb{X}$ of a directed graph $G=\left(V_{G}, E_{G}\right)$ (with set of edges $E_{G} \subset V_{G} \times V_{G}$ ), is a measurable function $\mathbb{X}: \Omega \rightarrow 2^{E_{G}}$ where $\Omega=(\Omega, \mathcal{A}, \mu)$ is a probability space, and $2^{E_{G}}$ is the powerset of $E_{G}$, identified with the set of all functions from $E_{G}$ to $\{0,1\}$ (with the product topology and the $\sigma$-algebra of its Borel sets). For each $x \in \Omega$, we identify $\mathbb{X}(x)$ with the subgraph of $G$ with vertices $V_{G}$ and edges $\mathbb{X}(x)$. Given $e \in E_{G}$, the set $\mathbb{X}_{e}:=\{x \in \Omega: e \in \mathbb{X}(x)\}$ represents the event that the random graph $\mathbb{X}$ contains the edge $e \in E_{G}$. The family $\left(\mathbb{X}_{e}\right)_{e \in E_{G}}$ determines $\mathbb{X}$ putting: $\mathbb{X}(x)=\left\{e \in E_{G}: x \in \mathbb{X}_{e}\right\}$. So a random subgraph of $G$ can be equivalently defined as a function from $E_{G}$ to $2^{\Omega}$ assigning to each $e \in E_{G}$ a measurable subset $\mathbb{X}_{e}$ of $\Omega$.

As in classic percolation theory, we wish to estimate the probability that $\mathbb{X}$ contains an infinite path, in terms of a parameter $\lambda$ that bounds from below the probability $\mu\left(\mathbb{X}_{e}\right)$ that an edge $e$ belongs to $\mathbb{X}$. Note that it is not a priori obvious that the existence of an infinite path has a well-defined probability, since it corresponds to the uncountable union of the sets $\bigcap_{k \in \mathbb{N}} \mathbb{X}_{i_{k}, i_{k+1}}$ over all strictly increasing sequences $i: \mathbb{N} \rightarrow \mathbb{N}$. However, it turns out that it belongs to the $\mu$-completion of the $\sigma$-algebra generated by the $\mathbb{X}_{i, j}$. It has to be noticed that the analogy with classic bond percolation is only formal, the main difference being that in the usual percolation models (see for instance G:99|) the events $\mathbb{X}_{i, j}$ are supposed independent, whereas in the present case the probability distribution is completely general, i.e. we do not impose any restriction on the events $\mathbb{X}_{i, j}$, and on the probability space $\Omega$.

Problem 1 has been originally proposed by P. Erdős and A. Hajnal in EH:64, and an answer was given by D. H. Fremlin and M. Talagrand in [FT:85], where other related and more general problems are also considered. In particular they show that the threshold for the existence of infinite paths is $\lambda=1 / 2$, under the assumption that the probability space $(\Omega, \mathcal{A}, \mu)$ is $[0,1]$ equipped with the Lebesgue measure (although the extension to a general probability space should not be difficult). One of the main goals of this paper is to present a general method, different from the one in FT:85, which in particular allows us to recover the same result as in [FT:85] (see Theorem 4.5). Our approach relies on the reduction to the following dual problem:

Problem 2. Given a directed graph $F$, determine the minimal $\lambda_{c}$ such that, whenever $\inf _{e \in \mathbb{N}^{(2)}} \mu\left(\mathbb{X}_{e}\right)>\lambda_{c}$, there is a graph morphism $f: \mathbb{X}(x) \rightarrow F$ for some $x \in \Omega$.

Problem 1 can be reformulated in this setting by letting $F$ be the graph $\left(\omega_{1},>\right)$ where $\omega_{1}$ is the first uncountable ordinal. This depends on the fact that a subgraph $H$ of $\left(\mathbb{N}, \mathbb{N}^{(2)}\right)$ does not contain an infinite path if and only if it admits a rank function with values in $\omega_{1}$. Therefore, if a random subgraph $\mathbb{X}$ of $\left(\mathbb{N}, \mathbb{N}^{(2)}\right)$ has no infinite paths, it is defined a $\mu$-measurable map $\varphi: \Omega \rightarrow \omega_{1}^{\mathbb{N}}$ where $\varphi(x)(i)$ is the rank of the vertex $i \in \mathbb{N}$ in the graph $\mathbb{X}(x)$. It turns out that $\varphi_{\#}(\mu)$ is a compactly supported Borel measure on $\omega_{1}^{\mathbb{N}}$, and that $\varphi\left(\mathbb{X}_{i, j}\right) \subseteq A_{i, j}:=\left\{x \in \omega_{1}^{\mathbb{N}}: x_{i}>x_{j}\right\}$. As a consequence, in 
the determination of the threshold for existence of infinite paths

$$
\lambda_{c}:=\sup \left\{\inf _{(i, j) \in \mathbb{N}^{(2)}} \mu\left(\mathbb{X}_{i, j}\right): \mathbb{X} \text { random graph without infinite paths }\right\}
$$

we can set $\Omega=\omega_{1}^{\mathbb{N}}, \mathbb{X}_{i, j}=A_{i, j}$, and reduce to the variational problem on the convex set $\mathcal{M}_{c}^{1}\left(\omega_{1}^{\mathbb{N}}\right)$ of compactly supported probability measures on $\omega_{1}^{\mathbb{N}}$ :

$$
\lambda_{c}=\sup _{m \in \mathcal{M}_{c}^{1}\left(\omega_{1}^{\mathbb{N}}\right)} \inf _{(i, j) \in \mathbb{N}^{(2)}} m\left(A_{i, j}\right) .
$$

As a next step, we show that in (1.2) we can equivalently take the supremum in the smaller class of all the compactly supported exchangeable measures on $\omega_{1}^{\mathbb{N}}$ (see Appendix $\mathrm{B}$ and references therein for a precise definition). Thanks to this reduction, we can explicitly compute $\lambda_{c}=1 / 2$ ( Theorem 4.5). We note that the supremum in (1.2) is not attained, which implies that for $\mu\left(\mathbb{X}_{i, j}\right) \geq 1 / 2$ infinite paths occurs with positive probability.

In Section 5, we consider again Problem 2 and we give a complete solution when $F$ is a finite graph, showing in particular that

$$
\lambda_{c}=\sup _{\lambda \in \Sigma_{F}} \sum_{(a, b) \in E_{F}} \lambda_{a} \lambda_{b}
$$

where $\Sigma_{F}$ is the set of all sequences $\left\{\lambda_{a}\right\}_{a \in V_{F}}$ with values in $[0,1]$ and such that $\sum_{a \in V_{F}} \lambda_{a}=1$. By the appropriate choice of $F$ we can determine the threholds for the existence of paths of a given finite length (Section 3 and Remark 5.2), or for the property of having chromatic number $\geq n$ (Section $6)$.

We can consider Problems 1 and 2 for a random subgraph $\mathbb{X}$ of an arbitrary directed graph $G$, not necessarily equal to $\left(\mathbb{N}, \mathbb{N}^{(2)}\right)$. However, it can be shown that, if we replace $\left(\mathbb{N}, \mathbb{N}^{(2)}\right)$ with a finitely branching graph $G$ (such as a finite dimensional network), the probability that $\mathbb{X}$ has an infinite path may be zero even if $\inf _{e \in E_{G}} \mu\left(\mathbb{X}_{e}\right)$ is arbitrarily close to 1 ( Proposition 4.8). Another variant is to consider subgraphs of $\mathbb{R}^{(2)}$ rathen than $\mathbb{N}^{(2)}$ but it turns out that this makes no difference in terms of the threshold for having infinite paths in random subgraphs (Remark 4.9).

In Section 6 we fix again $G=\left(\mathbb{N}, \mathbb{N}^{(2)}\right)$ and we ask if a random subgraph $\mathbb{X}$ of $G$ contains an infinite clique, i.e. a copy of $G$ itself. More generally we consider the following problem.

Problem 3. Let $(\Omega, \mathcal{A}, \mu)$ be a probability space. Let $\lambda>0$ and for all $\left(i_{1}, \ldots, i_{k}\right) \in \mathbb{N}^{(k)}$, let $\mathbb{X}_{i_{1}, \ldots, i_{k}}$ be a measurable subset of $X$ with $\mu\left(\mathbb{X}_{i_{1}, \ldots, i_{k}}\right) \geq$ $\lambda$. Is there an infinite set $J \subset \mathbb{N}$ such that $\bigcap_{\left(i_{1}, \ldots, i_{k}\right) \in J^{(k)}} \mathbb{X}_{i_{1}, \ldots, i_{k}}$ is nonempty?

This problem is a random version of the classical Ramsey theorem R:28 (we refer to [GP:73, PR:05], and references therein, for various generalization of Ramsey theorem). Clearly Ramsey theorem implies that the answer to Problem 3 is positive when $\Omega$ is finite. Moreover it can be shown that the answer remains positive when $\Omega$ is countable (Example 6.3). However when $\Omega=[0,1]$ (with the Lebesgue measure) the probability that $\mathbb{X}$ contains an infinite clique may be zero even when $\inf _{e \in E_{G}} \mu\left(\mathbb{X}_{e}\right)$ is arbitrarily close to 1 
(see Example 6.2). We will show that Problem 3 has a positive answer if the indicator functions of the sets $\mathbb{X}_{i_{1}, \ldots, i_{k}}$ all belong to a compact subset of $L^{1}(\Omega, \mu)$ (see Theorem 6.5).

Our original motivation for the above problems came from the following situation. Suppose we are given a space $E$ and a certain family $\Omega$ of sequences on $E$ (e.g., minimizing sequences of a functional, or orbits of a discrete dynamical system, etc). A typical general problem asks for existence of a sequence in the family $\Omega$, that admits a subsequence with a prescribed property. One approach to it is by means of measure theory. The archetypal situation here come from recurrence theorems: one may ask if there exists a subsequence which belongs frequently to a given subset $C$ of the "phase" space $\Omega$ (we refer to such sequences as " $C$-recurrent orbits"). If we consider the set $\mathbb{X}_{i}:=\left\{x \in \Omega: x_{i} \in C\right\}$, then a standard sufficient condition for existence of $C$-recurrent orbits is $\mu\left(\mathbb{X}_{i}\right) \geq \lambda>0$, for some probability measure $\mu$ on $\Omega$. In fact is easy to check that the set of $C$-recurrent orbits has measure at least $\lambda$ by an elementary version of a Borel-Cantelli lemma (see Proposition 6.1). This is indeed the existence argument in the Poincaré Recurrence Theorem for measure preserving transformations. A more subtle question arises when one looks for a subsequence satisfying a given relation between two successive (or possibly more) terms: given a subset $R$ of $E \times E$ we look for a subsequence $x_{i_{k}}$ such that $\left(x_{i_{k}}, x_{i_{k+1}}\right) \in R$ for all $k \in \mathbb{N}$. As before, we may consider the subset of $\Omega$, with double indices $i<j, \mathbb{X}_{i, j}:=\left\{x \in \Omega:\left(x_{i}, x_{j}\right) \in R\right\}$ and we are then led to Problem $\mathbb{1}$.

\section{Notations}

We follow the set-theoretical convention of identifying a natural number $p$ with the set $\{0,1, \ldots, p-1\}$ of its predecessors. More generally an ordinal number $\alpha$ coincides with the set of its predecessors. With these conventions the set of natural numbers $\mathbb{N}$ coincides with the least infinite ordinal $\omega$. As usual $\omega_{1}$ denotes the first uncountable ordinal, namely the set of all countable ordinals.

Given two sets $X, Y$ we denote by $X^{Y}$ the set of all functions from $Y$ to $X$. If $X, Y$ are linearly ordered we denote by $X^{(Y)}$ the set of all increasing functions from $Y$ to $X$. In particular $\mathbb{N}^{(p)}$ (with $p \in \mathbb{N}$ ) is the set of all increasing $p$-tuples from $\mathbb{N}$, where a $p$-tuple $\boldsymbol{i}=\left(i_{0}, \ldots, i_{p-1}\right)$ is a function $\boldsymbol{i}: p \rightarrow \mathbb{N}$. The case $p=2$, with the obvious identifications, takes the form $\mathbb{N}^{(2)}=\left\{(i, j) \in \mathbb{N}^{2}: i<j\right\}$.

Any function $f: X \rightarrow X$ induces a function $f_{*}: X^{Y} \rightarrow X^{Y}$ by $f(u)=$ $f \circ u$. On the other hand a function $f: Y \rightarrow Z$ induces a function $f^{*}: X^{Z} \rightarrow$ $X^{Y}$ by $f^{*}(u)=u \circ f$. In particular if $\mathbf{S}: \mathbb{N} \rightarrow \mathbb{N}$ is the successor function, $\mathrm{S}^{*}: X^{\mathbb{N}} \rightarrow X^{\mathbb{N}}$ is the shift map.

We let $\mathfrak{S}_{c}(\mathbb{N}), \operatorname{Inj}(\mathbb{N}), \operatorname{Incr}(\mathbb{N}) \subset \mathbb{N}^{\mathbb{N}}$ be the families of maps $\sigma: \mathbb{N} \rightarrow$ $\mathbb{N}$ which are compactly supported permutations injective functions and strictly increasing functions, respectively. Note that with the above conventions $\operatorname{Incr}(\mathbb{N})=\mathbb{N}^{(\omega)}$.

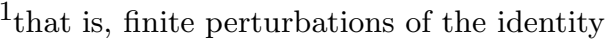


Given a measurable function $\psi: X \rightarrow Y$ between two measurable spaces and given a measure $m$ on $X$, we denote as usual by $\psi_{\#}(m)$ the induced measure on $Y$.

Given a compact metric space $\Lambda$, the space $\mathcal{M}\left(\Lambda^{\mathbb{N}}\right)$ of Borel measures on $\Lambda^{\mathbb{N}}$ can be identified with $C\left(\Lambda^{\mathbb{N}}\right)^{*}$, i.e. the dual of the Banach space of all continuous functions on $\Lambda^{\mathbb{N}}$. By the Banach-Alaoglu theorem the subset $\mathcal{M}^{1}\left(\Lambda^{\mathbb{N}}\right) \subset \mathcal{M}\left(\Lambda^{\mathbb{N}}\right)$ of probability measures is a compact (metrizable) subspace of $C\left(\Lambda^{\mathbb{N}}\right)^{*}$ endowed with the weak* topology.

Given $\sigma: \mathbb{N} \rightarrow \mathbb{N}$ we have $\sigma^{*}: \Lambda^{\mathbb{N}} \rightarrow \Lambda^{\mathbb{N}}$ and $\sigma_{\#}^{*}: \mathcal{M}^{1}\left(\Lambda^{\mathbb{N}}\right) \rightarrow \mathcal{M}^{1}\left(\Lambda^{\mathbb{N}}\right)$. To simplify notations we also write $\sigma \cdot m$ for $\sigma_{\#}^{*} m$. Note the contravariance of this action:

$$
\theta \cdot \sigma \cdot m=(\sigma \circ \theta) \cdot m .
$$

Similarly given $r \in \mathbb{N}$ and $\iota \in \mathbb{N}^{(r)}$, we have $\iota_{\#}^{*}: \mathcal{M}^{1}\left(\Lambda^{\mathbb{N}}\right) \rightarrow \mathcal{M}^{1}\left(\Lambda^{r}\right)$ and we define $\iota \cdot m=\iota_{\#}^{*}(m)$.

Given a family $\mathcal{F} \subset \mathbb{N}^{\mathbb{N}}$, we say that $m$ is $\mathcal{F}$-invariant if $\sigma \cdot m=m$ for all $\sigma \in \mathcal{F}$.

\section{Finite PATHS In RANDOM SUBGRAPHS}

As a preparation for the study of infinite paths (Problem 1) we first consider the case of finite paths. The following example shows that there are random subgraphs $\mathbb{X}$ of $\left(\mathbb{N}, \mathbb{N}^{(2)}\right)$ such that inf ${ }_{e \in \mathbb{N}^{(2)}} \mathbb{X}_{e}$ is arbitrarily close to $1 / 2$, and yet $\mathbb{X}$ has probability zero of having infinite paths.

Example 3.1. Let $p \in \mathbb{N}$ and let $\Omega=p^{\mathbb{N}}$ with the Bernoulli probability measure $\mu=B_{(1 / p, \ldots, 1 / p)}$. For $i<j$ in $\mathbb{N}$ let $\mathbb{X}_{i, j}=\left\{x \in p^{\mathbb{N}}: x_{i}>x_{j}\right\}$. Then $\mu\left(\mathbb{X}_{i, j}\right)=\frac{1}{2}\left(1-\frac{1}{p}\right)$ for all $(i, j) \in \mathbb{N}^{(2)}$ and yet for each $x \in \Omega$ the graph $\mathbb{X}(x)=\left\{(i, j) \in \mathbb{N}^{(2)}: x_{i}>x_{j}\right\}$ has no paths of length $\geq p$ (where the length of a path is the number of its edges).

We will next show that the bounds in Example 3.1 are optimal. We need:

Lemma 3.2. Let $p \in \mathbb{N}$ and let $m \in \mathcal{M}^{1}\left(p^{\mathbb{N}}\right)$. Let

$$
A_{i, j}:=\left\{x \in p^{\mathbb{N}}: x_{i}>x_{j}\right\} .
$$

Then

$$
\inf _{(i, j) \in \mathbb{N}^{(2)}} m\left(A_{i, j}\right) \leq \frac{1}{2}\left(1-\frac{1}{p}\right)
$$

Proof. The proof is a reduction to the case of exchangeable measures (see Appendix $\mathbb{B})$. Note that if $\sigma \in \operatorname{Incr}(\mathbb{N})$, then $(\sigma \cdot m)\left(A_{i, j}\right)=m\left(A_{\sigma(i), \sigma(j)}\right)$. Hence, replacing $m$ with $\sigma \cdot m$ in (3.2) can only increase the infimum, as it is equivalent to the infimum of $m\left(A_{i, j}\right)$ over a subset of $\mathbb{N}^{(2)}$. By Theorem B.8 we can then assume that $m$ is asymptotically exchangeable, so that in particular the sequence $m_{k}=\mathrm{S}^{k} \cdot m$ converges, in the weak* topology, to an exchangeable measure $m^{\prime} \in \mathcal{M}^{1}\left(p^{\mathbb{N}}\right)$. Since $p$ is finite, the sets $A_{i, j}$ are clopen, and therefore $\lim _{k \rightarrow \infty} m_{k}\left(A_{i, j}\right)=m^{\prime}\left(A_{i, j}\right)=m^{\prime}\left(A_{0,1}\right)$. Noting that 
$m_{k}\left(A_{i, j}\right)=m\left(A_{i+k, j+k}\right)$, it follows that

$$
\begin{aligned}
\inf _{(i, j) \in \mathbb{N}^{(2)}} m\left(A_{i, j}\right) & \leq \lim _{k \rightarrow \infty} m_{k}\left(A_{0,1}\right) \\
& =m^{\prime}\left(A_{0,1}\right) \\
& =\frac{1}{2}\left(1-m^{\prime}\left\{x: x_{0}=x_{1}\right\}\right) \\
& \leq \frac{1}{2}\left(1-\frac{1}{p}\right)
\end{aligned}
$$

where the latter inequality follows from Corollary B.11.

Theorem 3.3. Let $(\Omega, \mathcal{A}, \mu)$ be a probability space and let $\mathbb{X}: \Omega \rightarrow 2^{E_{G}}$ be a random subgraph of $G:=\left(\mathbb{N}, \mathbb{N}^{(2)}\right)$. Consider the set

$$
P:=\{x \in \Omega: \mathbb{X}(x) \text { has a path of length } \geq p\} .
$$

Assume $\inf _{e \in \mathbb{N}^{(2)}} \mu\left(\mathbb{X}_{e}\right)>\frac{1}{2}\left(1-\frac{1}{p}\right)$. Then $\mu(P)>0$.

A different proof of this result has been given in [FT:85, 3F] (when the probability space $\Omega$ is $[0,1]$ equipped with the Lebesgue measure).

Proof. Suppose for a contradiction that $\mu(P)=0$. We can then assume $P=\emptyset$ (otherwise replace $\Omega$ with $\Omega-P$ ). For $x \in \Omega$ let $\varphi(x): \mathbb{N} \rightarrow p$ assign to each $i \in \mathbb{N}$ the length of the longest path starting from $i$ in $\mathbb{X}(x)$. We thus obtain a function $\varphi: \Omega \rightarrow p^{\mathbb{N}}$ which is easily seen to be measurable (this is a special case of Lemma 4.3). Let $m=\varphi_{\#}(\mu) \in \mathcal{M}^{1}\left(p^{\mathbb{N}}\right)$. Since $\varphi\left(\mathbb{X}_{i, j}\right) \subset A_{i, j}$, we have $m\left(A_{i, j}\right) \geq \mu\left(\mathbb{X}_{i, j}\right) \geq 1 / 2\left(1-\frac{1}{p}\right)$ for all $i, j$, contradicting Lemma 3.2 .

Having determined the critical threshold $\lambda_{p}=\frac{1}{2}\left(1-\frac{1}{p}\right)$, it follows that if $\inf _{e \in \mathbb{N}^{(2)}} \mu\left(\mathbb{X}_{e}\right) \geq \lambda \geq \lambda_{p}$, the lower bound for $\mu(P)$ grows linearly with $\lambda$. More precisely we have:

Corollary 3.4. In the setting of Theorem 3.3, let $\lambda \in[0,1]$ and suppose that $\inf _{e \in \mathbb{N}^{(2)}} \mu\left(\mathbb{X}_{e}\right) \geq \lambda$. Then $\mu(P) \geq \frac{\lambda-\lambda_{p}}{1-\lambda_{p}}$ where $\lambda_{p}=\frac{1}{2}\left(1-\frac{1}{p}\right)$.

Proof. Suppose $\inf _{e \in \mathbb{N}^{(2)}} \mu\left(\mathbb{X}_{e}\right) \geq \lambda$. Consider the conditional probability $\mu(\cdot \mid \Omega-P) \in \mathcal{M}^{1}(\Omega)$. We have

$$
\begin{aligned}
\mu\left(\mathbb{X}_{e} \mid \Omega-P\right) & \geq \frac{\mu\left(\mathbb{X}_{e}\right)-\mu(P)}{1-\mu(P)} \\
& \geq \frac{\lambda-\mu(P)}{1-\mu(P)} .
\end{aligned}
$$

Clearly $\mu(P \mid \Omega-P)=0$. Applying Theorem 3.3 to $\mu(\cdot \mid \Omega-P)$ it then follows that $\frac{\lambda-\mu(P)}{1-\mu(P)} \leq \lambda_{p}$, or equivalently $\mu(P) \geq \frac{\lambda-\lambda_{p}}{1-\lambda_{p}}$.

\section{INFINITE PATHS}

By Theorem 3.3, if inf $\operatorname{e\in \mathbb {N}}^{(2)} \mu\left(\mathbb{X}_{i, j}\right) \geq 1 / 2$, then the random subgraph $\mathbb{X}$ of $\left(\mathbb{N}, \mathbb{N}^{(2)}\right)$ has arbitrarily long finite paths, namely for each $p$ there is $x \in \Omega$ (depending on $p$ ) such that $\mathbb{X}(x)$ has a path of length $\geq p$. We want to show that for some $x \in \Omega, \mathbb{X}(x)$ has an infinite path. To this aim it is not enough 
to find a single $x$ that works for all $p$. Indeed, $\mathbb{X}(x)$ could have arbitrarily long finite paths without having an infinite path. The existence of infinite paths can be neatly expressed in terms of the following definition.

Definition 4.1. Let $G$ be a countable directed graph and let $\omega_{1}$ be the first uncountable ordinal. We recall that the rank function $\varphi_{G}: V_{G} \rightarrow \omega_{1} \cup\{\infty\}$ of $G$ is defined as follows. For $i \in V_{G}$,

$$
\varphi_{G}(i)=\sup _{j:(i, j) \in E_{G}}\left(\varphi_{G}(j)+1\right) .
$$

This is a well defined countable ordinal if $G$ has no infinite paths starting at $i$. In the opposite case we set

$$
\varphi_{G}(i)=\infty
$$

where $\infty$ is a conventional value bigger than all the countable ordinals. For notational convenience we will take $\infty=\omega_{1}$ so that $\omega_{1} \cup\{\infty\}=\omega_{1} \cup\left\{\omega_{1}\right\}=$ $\omega_{1}+1$. Note that if $i$ is a leaf, $\varphi_{G}(i)=0$. Also note that $G$ has an infinite path if and only if $\varphi_{G}$ assumes the value $\infty$.

Given a random subgraph $\mathbb{X}: \Omega \rightarrow 2^{E_{G}}$ of $G$, we let $\varphi_{\mathbb{X}}(x)=\varphi_{\mathbb{X}(x)}$, namely $\varphi_{\mathbb{X}}(x)(i)$ is the rank of the vertex $i$ in the graph $\mathbb{X}(x)$. So $\varphi_{\mathbb{X}}$ is a map from $\Omega$ to $\left(\omega_{1}+1\right)^{V_{G}}$. It can also be considered as a map from $\Omega \times V_{G}$ to $\omega_{1}+1$ by writing $\varphi_{\mathbb{X}}(x, i)$ instead of $\varphi_{\mathbb{X}}(x)(i)$.

Remark 4.2. We have $\varphi_{\mathbb{X}}(x, i)=\varphi_{\omega_{1}}(x, i)$ where $\varphi_{\alpha}: \Omega \rightarrow\left(\omega_{1}+1\right)^{V_{G}}$ is the truncation $\varphi_{\alpha}:=\min (\varphi, \alpha)$, that we can equivalently define by induction on $\alpha \leq \omega_{1}$ as follows.

$$
\begin{aligned}
& \varphi_{0}(x, i)=0 \\
& \varphi_{\alpha}(x, i)=\sup \left\{\varphi_{\beta}(x, j)+1: \beta<\alpha,(i, j) \in \mathbb{X}(x)\right\}
\end{aligned}
$$

The above representation will be of use in the following lemma in connection to measurability properties of the map $\varphi$.

Lemma 4.3. Let $G$ be a countable directed graph, let $(\Omega, \mathcal{A}, \mu)$ be a probability space and let $\mathbb{X}: \Omega \rightarrow 2^{E_{G}}$ be a random subgraph of $G$.

(1) The set $P:=\{x \in \Omega: \mathbb{X}(x)$ has an infinite path $\}$ is $\mu$-measurable.

(2) For all $\alpha \leq \omega_{1}$ and $i \in V_{G}$, the set $\left\{x \in \Omega: \varphi_{\mathbb{X}}(x, i)=\alpha\right\}$ is $\mu$-measurable.

(3) $\varphi_{\mathbb{X}}: \Omega \rightarrow\left(\omega_{1}+1\right)^{V_{G}}$ is $\mu$-measurable and its restriction to $\Omega-P$ is essentially bounded, namely for some $\alpha_{0}<\omega_{1}$ it takes values in $\alpha_{0}^{V_{G}}$ outside of a $\mu$-null set.

Proof. Since taking the supremum over a countable set preserves measurability, from Remark 4.2 it follows that for all $i \in V_{G}$ and $\alpha<\omega_{1}$ the sets $\left\{x: \varphi_{\mathbb{X}}(x, i)=\alpha\right\}$ are measurable. We will show that $\left\{x: \varphi_{\mathbb{X}}(x, i)=\omega_{1}\right\}$ is $\mu$-measurable, namely it is the union of a measurable set and a $\mu$-null set. Fix $i \in V_{G}$. The sequence of values $\mu\left(\left\{x: \varphi_{\mathbb{X}}(x, i) \leq \beta\right\}\right)$ is increasing with respect to the countable ordinal $\beta$ and uniformly bounded by $1=\mu(\Omega)$, therefore it is stationary at some finite value. So there is $\alpha_{0}<\omega_{1}$ such that

$$
\mu\left(\left\{x \in \Omega: \varphi_{\mathbb{X}}(x, k)=\beta\right\}\right)=0 \quad \text { for } \alpha_{0} \leq \beta<\omega_{1} .
$$

It follows that $\left\{x: \varphi_{\mathbb{X}}(x, i)=\omega_{1}\right\}$ is $\mu$-measurable and $\varphi_{\mathbb{X}}$ is $\mu$-measurable. Since $P=\cup_{i}\left\{x: \varphi_{\mathbb{X}}(x, i)=\omega_{1}\right\}$, we have that $P$ is $\mu$-measurable,too. 
Given an ordinal $\alpha$, we put on $\alpha$ the topology generated by the open intervals. Note that a non-zero ordinal is compact if and only if it is a successor ordinal, and it is metrizable if and only if it is countable. Let $\mathcal{M}_{c}\left(\omega_{1}^{\mathbb{N}}\right)$ be the set of compactly supported Borel measures on $\omega_{1}^{\mathbb{N}}$, namely the measures with support in $\alpha_{0}{ }^{\mathbb{N}}$ for some $\alpha_{0}<\omega_{1}$. The following Lemma reduces to Lemma 3.2 if $\alpha_{0}$ is finite.

Lemma 4.4. Let $m \in \mathcal{M}_{c}\left(\omega_{1}^{\mathbb{N}}\right)$ be a non-zero measure with compact support. Let

$$
A_{i, j}:=\left\{x \in \omega_{1}^{\mathbb{N}}: x_{i}>x_{j}\right\}
$$

Then

$$
\inf _{(i, j) \in \mathbb{N}^{(2)}} m\left(A_{i, j}\right)<\frac{m\left(\omega_{1}^{\mathbb{N}}\right)}{2} .
$$

Proof. With no loss of generality we can assume that $m \in \mathcal{M}^{1}\left(\omega_{1}^{\mathbb{N}}\right)$, i.e. $m\left(\omega_{1}^{\mathbb{N}}\right)=1$. We divide the proof into four steps.

Step 1. Letting $\partial \omega_{1}$ be the derived set of $\omega_{1}$, that is the subset of all countable limit ordinals, we can assume that

$$
m\left(\left\{x: x_{i} \in \partial \omega_{1}\right\}\right)=0 \quad \forall i \in \mathbb{N} .
$$

Indeed, it is enough to observe that the left-hand side of equation (4.2) can only increase if we replace $m$ with $s_{\#}(m)$, where $s: \omega_{1} \rightarrow \omega_{1} \backslash \partial \omega_{1}$ is the successor map sending $\alpha<\omega_{1}$ to $\alpha+1$, and $s_{\#}(m)=\left(s_{*}\right)_{\#}$, namely $s_{\#}(m)(X):=m\left(\left\{x \in \omega_{1}^{\mathbb{N}}: s \circ x \in X\right\}\right)$.

Step 2. Since the support of $m$ is contained in $\alpha_{0}^{\mathbb{N}}$, for some ordinal $\alpha_{0}<\omega_{1}$, thanks to Theorem B.8 we can assume that $m$ is asymptotically exchangeable, i.e. the sequence $m_{k}=\mathrm{S}^{k} \cdot \sigma \cdot m$ converges, in the weak* topology, to an exchangeable measure $m^{\prime} \in \mathcal{M}^{1}\left(\omega_{1}^{\mathbb{N}}\right)$, with support in $\alpha_{0}^{\mathbb{N}}$, for all $\sigma \in \omega^{(\omega)}$. Note however that, unless $\alpha_{0}$ is finite, we cannot conclude that $\lim _{k \rightarrow \infty} m_{k}\left(A_{i, j}\right)=m^{\prime}\left(A_{i, j}\right)$ since the sets $A_{i, j}=\left\{x \in \omega_{1}^{\mathbb{N}}: x_{i}>x_{j}\right\}$ are not clopen.

Step 3. We shall prove by induction on $\alpha<\omega_{1}$ that

$$
\liminf _{(i, j) \rightarrow+\infty} m\left(\left\{x: x_{j}<x_{i} \leq \alpha\right\}\right) \leq m^{\prime}\left(\left\{x: x_{1}<x_{0} \leq \alpha\right\}\right) .
$$

Indeed, for $\alpha=0$ we have $\left\{x: x_{j}<x_{i} \leq 0\right\}=\emptyset$, and (4.3) holds.

As inductive step, let us assume that (4.3) holds for all $\alpha<\beta<\omega_{1}$, and we distinguish whether $\beta$ is a successor or a limit ordinal.

In the former case let $\beta=\alpha+1$. For $(i, j) \rightarrow+\infty$ (with $i<j$ ) we have:

$$
\begin{aligned}
m\left(\left\{x_{j}<x_{i} \leq \beta\right\}\right) & =m\left(\left\{x_{j}<x_{i} \leq \alpha\right\}\right)+m\left(\left\{x_{j} \leq \alpha, x_{i}=\beta\right\}\right) \\
& \leq m^{\prime}\left(\left\{x_{1}<x_{0} \leq \alpha\right\}\right)+m^{\prime}\left(\left\{x_{1} \leq \alpha, x_{0}=\beta\right\}\right)+o(1) \\
& =m^{\prime}\left(\left\{x_{1}<x_{0} \leq \beta\right\}\right)+o(1),
\end{aligned}
$$

where we used the induction hypothesis, and the fact that $\left\{x_{j} \leq \alpha, x_{i}=\beta\right\}$ is clopen.

Let us now assume that $\beta$ is a limit ordinal and let $i \in \mathbb{N}$. We have

$$
\bigcap_{\alpha<\beta}\left\{x: \alpha<x_{i}<\beta\right\}=\emptyset
$$


so for all $\varepsilon>0$ there exists $\alpha<\beta$ such that

$$
m^{\prime}\left(\left\{\alpha<x_{i}<\beta\right\}\right)<\varepsilon .
$$

Since $m^{\prime}$ is exchangeable, we can choose the same $\alpha$ for every $i$. Moreover by assumption $m\left(\left\{x_{i}=\beta\right\}\right)=0$ for every $i \in \mathbb{N}$. Hence there exists $\alpha \leq \alpha_{i}<\beta$ such that

$$
m\left(\left\{\alpha_{i} \leq x_{i} \leq \beta\right\}\right)<\varepsilon .
$$

Given $i<j$, distinguishing the relative positions of $x_{i}, x_{j}$ with respect to $\alpha$ and $\alpha_{i}$ we have:

$$
\begin{aligned}
\left\{x_{j}<x_{i} \leq \beta\right\} \subseteq & \left\{x_{j}<x_{i} \leq \alpha\right\} \\
& \cup\left\{x_{j} \leq \alpha<x_{i} \leq \beta\right\} \\
& \cup\left\{\alpha<x_{j} \leq \alpha_{i}\right\} \\
& \cup\left\{\alpha_{i}<x_{i} \leq \beta\right\} .
\end{aligned}
$$

which gives

$$
\begin{aligned}
m\left(\left\{x_{j}<x_{i} \leq \beta\right\}\right) \leq & m\left(\left\{x_{j}<x_{i} \leq \alpha\right\}\right) \\
& +m\left(\left\{x_{j} \leq \alpha<x_{i} \leq \beta\right\}\right) \\
& +m\left(\left\{\alpha<x_{j} \leq \alpha_{i}\right\}\right) \\
& +m\left(\left\{\alpha_{i}<x_{i} \leq \beta\right\}\right) .
\end{aligned}
$$

Since $\left\{x_{j} \leq \alpha<x_{i} \leq \beta\right\}$ and $\left\{\alpha<x_{j} \leq \alpha_{i}\right\}$ are both clopen, we can approximate their $m$-measure by their $m^{\prime}$-measure. So we have:

$$
\begin{aligned}
m\left\{x_{j} \leq \alpha<x_{i} \leq \beta\right\}= & m^{\prime}\left(\left\{x_{1} \leq \alpha<x_{0} \leq \beta\right\}\right)+o(1) \\
& \text { for }(i, j) \rightarrow \infty
\end{aligned}
$$

and

$$
\begin{aligned}
m\left(\left\{\alpha<x_{j} \leq \alpha_{i}\right\}\right)= & m^{\prime}\left(\left\{\alpha<x_{1} \leq \alpha_{i}\right\}\right)+o(1) \\
& \text { for } j \rightarrow \infty,
\end{aligned}
$$

where we used Remark B.7 to allow $j \rightarrow \infty$ keeping $i$ fixed. Now note that by the choice of $\alpha$, we have $m^{\prime}\left(\left\{\alpha<x_{1} \leq \alpha_{i}\right\}\right)<\varepsilon$, and by induction hypothesis $\liminf (i, j) \rightarrow+\infty m\left(\left\{x_{j}<x_{i} \leq \alpha\right\}\right)<m^{\prime}\left(\left\{x_{1}<x_{0} \leq \beta\right\}\right)$. Hence, from (4.4) we obtain:

$$
\begin{aligned}
\liminf _{(i, j) \rightarrow+\infty} m\left(\left\{x_{j}<x_{i} \leq \beta\right\}\right) \leq & m^{\prime}\left(\left\{x_{1}<x_{0} \leq \alpha\right\}\right) \\
& +m^{\prime}\left(\left\{x_{1} \leq \alpha<x_{0} \leq \beta\right\}\right) \\
& +\varepsilon+\varepsilon
\end{aligned}
$$

Therefore,

$$
\liminf _{(i, j) \rightarrow+\infty} m\left(\left\{x_{j}<x_{i} \leq \beta\right\}\right) \leq m^{\prime}\left(\left\{x_{1}<x_{0} \leq \beta\right\}\right)+2 \varepsilon
$$

Inequality (4.3) is then proved for all $\alpha<\omega_{1}$.

Step 4. We now conclude the proof of the theorem. From (4.3) it follows

$$
\inf _{(i, j) \in \mathbb{N}^{(2)}} m\left(A_{i, j}\right) \leq m^{\prime}\left(\left\{x: x_{1}<x_{0}\right\}\right)=\frac{1}{2}\left(1-m^{\prime}\left(\left\{x: x_{1}=x_{0}\right\}\right)\right)<\frac{1}{2} .
$$


where we used the fact the $m^{\prime}$ is exchangeable and Corollary B.10.

Theorem 4.5. Let $(\Omega, \mathcal{A}, \mu)$ be a probability space and let $\mathbb{X}: \Omega \rightarrow 2^{E_{G}}$ be a random subgraph of $G:=\left(\mathbb{N}, \mathbb{N}^{(2)}\right)$. Consider the set

$$
P:=\{x \in \Omega: \mathbb{X}(x) \text { has an infinite path }\} .
$$

Assume $\inf _{e \in \mathbb{N}^{(2)}} \mu\left(\mathbb{X}_{e}\right) \geq \frac{1}{2}$. Then $\mu(P)>0$.

As observed in the Introduction, we recall that this result follows from [FT:85, 4D], when $\Omega=[0,1]$ with the Lebesgue measure.

Proof. Suppose for a contradiction $\mu(P)=0$. We can then assume $P=\emptyset$ (replacing $\Omega$ with $\Omega-P$ ). Hence the rank function $\varphi:=\varphi_{\mathbb{X}}: \Omega \rightarrow\left(\omega_{1}+1\right)^{\mathbb{N}}$ takes values in $\omega_{1}^{\mathbb{N}}$. Let $m=\varphi_{\#}(\mu) \in \mathcal{M}^{1}\left(\omega_{1}^{\mathbb{N}}\right)$. Note that $\varphi\left(\mathbb{X}_{i, j}\right) \subset A_{i . j}:=$ $\left\{x \in p^{\mathbb{N}}: x_{i}>x_{j}\right\}$. Hence $m\left(A_{i, j}\right) \geq \mu\left(\mathbb{X}_{i, j}\right) \geq 1 / 2$ for all $(i, j) \in \mathbb{N}^{(2)}$. This contradicts Lemma 4.4.

Remark 4.6. Note that the bound $1 / 2$ is optimal by Example 3.1.

Reasoning as in Corollary 3.4 we obtain:

Corollary 4.7. Let $0 \leq \lambda<1$. If $\inf _{e \in \mathbb{N}^{(2)}} \mu\left(\mathbb{X}_{e}\right) \geq \lambda$, then $\mu(P)>\frac{\lambda-1 / 2}{1-1 / 2}$.

Note that if we replace $\left(\mathbb{N}, \mathbb{N}^{(2)}\right)$ with a finitely branching countable graph $G$, then the threshold for the existence of infinite paths becomes 1 , namely we cannot ensure the existence of infinite paths even if each edge of $G$ belongs to the random subgraph $\mathbb{X}$ with probability very close to 1 . In fact, the following more general result holds:

Proposition 4.8. Let $G=\left(V_{G}, E_{G}\right)$ be graph admitting a coloring function $c: E_{G} \rightarrow \mathbb{N}$ such that each infinite path in $G$ meets all but finitely many colours (it is easy to see that a finitely branching countable graph $G$ has this property). Then for every $\varepsilon>0$ there is a probability space $(\Omega, \mathcal{A}, \mu)$ and a random subgraph $\mathbb{X}: \Omega \rightarrow 2^{E_{G}}$ of $G$ such that for all $x \in \Omega, \mathbb{X}(x)$ has no infinite paths, and yet $\mu\left(\mathbb{X}_{e}\right)>1-\varepsilon$ for all $e \in E_{G}$.

Proof. Let $\left(Z_{n}\right)_{n \in \mathbb{N}}$ be a disjoint family of infinite subsets of $\mathbb{N}$. Let $\mu$ be a probability measure on $\Omega:=\mathbb{N}$ with $\mu(\{n\})<\varepsilon$ for every $n$. Given $n \in \Omega$ let $\mathbb{X}(n)$ be the subgraph of $G$ (with vertices $V_{G}$ ) containing all edges $e \in E_{G}$ of colour $c(e) \notin Z_{n}$. Given $e \in E_{G}$ there is at most one $n$ such that $c(e) \in Z_{n}$. Hence clearly $\mu\left(\mathbb{X}_{e}\right) \geq 1-\varepsilon$, and yet $\mathbb{X}(n)$ has no infinite paths for any $n \in \Omega$.

Remark 4.9. It is natural to ask whether the answer to Problem 1 changes if we substitute $\mathbb{N}$ with the set of the real numbers. Since $\mathbb{N} \subset \mathbb{R}$, the probability threshold for the existence of infinite paths can only decrease, but the following example shows that it still equals $1 / 2$. Let $\Omega=[0,1]^{\mathbb{R}}$ equipped with the product Lebesgue measure $\mathcal{L}$, let $\varepsilon>0$, and let

$$
\mathbb{X}_{i, j}:=\left\{x \in \Omega: x_{i}>x_{j}+\varepsilon\right\},
$$

for all $i<j \in \mathbb{R}$. The assertion follows observing that $\mathcal{L}\left(\mathbb{X}_{i, j}\right)=(1-\varepsilon)^{2} / 2$ for all $i<j \in \mathbb{R}$, and

$$
\bigcap_{i \in\{1, \ldots, N\}} \mathbb{X}_{n_{i}, n_{i+1}}=\emptyset
$$


whenever $n_{i}$ is a strictly increasing sequence of real numbers, and $N>1 / \varepsilon$.

\section{Threshold FUNCTIONS FOR GRAPH MORPHISMS}

Definition 5.1. Let $F$ and $G$ be directed graphs. A graph morphism $\varphi: G \rightarrow F$ is a map $\varphi: V_{G} \rightarrow V_{F}$ such that $(\varphi(a), \varphi(b)) \in E_{F}$ for all $(a, b) \in E_{G}$. We write $G \rightarrow F$ if there is a graph morphism from $G$ to $F$.

The results of the previous sections were implicitly based on following observation:

Remark 5.2. Let $G$ be a directed graph.

(1) $G$ has a path of length $\geq p$ if and only if $G \not \rightarrow\left(p, p^{(2)}\right)$.

(2) $G$ has an infinite path if and only if $G \not \rightarrow\left(\omega_{1}, \omega_{1}^{(2)}\right)$.

This suggests to generalize the above results considering other properties of graphs that can be expressed in terms of non-existence of graph morphisms. Let us give the relevant definitions.

Definition 5.3. Given two directed graphs $F, G$ and given $i, j \in V_{G}$ let

$$
A_{i, j}(F, G):=\left\{u \in V_{F}^{V_{G}}:(u(i), u(j)) \in E_{F}\right\}
$$

and define the relative capacity of $F$ with respect to $G$ as

$$
c(F, G):=\sup _{m \in \mathcal{M}^{1}\left(V_{F}^{V_{G}}\right)} \inf _{(i, j) \in E_{G}} m\left(A_{i, j}(F, G)\right) \in[0,1] .
$$

Theorems 3.3 and 4.5 have the following counterpart.

Theorem 5.4. Let $F$ and $G$ be directed countable graphs, let $(\Omega, \mathcal{A}, \mu)$ be a probability space and let $\mathbb{X}: \Omega \rightarrow 2^{E_{G}}$ be a random subgraph of $G$. Let $P:=$ $\{x \in \Omega: \mathbb{X}(x) \not \supset F\}$. Assume $\inf _{e \in E_{G}} \mu\left(\mathbb{X}_{e}\right)>c(F, G)$. Then $\mu(P)>0$. Moreover there are examples in which $P$ is empty and $\inf _{e \in E_{G}} \mu\left(\mathbb{X}_{e}\right)$ is as close to $c(F, G)$ as required. So $c(F, G)$ is the threshold for non-existence of graph morphisms $f: \mathbb{X}(x) \rightarrow F$. To prove the second part it suffices to take $\Omega=V_{F}^{V^{G}}$ and $\mathbb{X}_{i, j}=A_{i, j}(F, G)$.

Proof. Suppose for a contradiction $\mu(P)=0$. We can then assume $P=\emptyset$ (replacing $\Omega$ with $\Omega-P$ ). Hence for each $x \in \Omega$ there is a graph morphism $\varphi(x): \mathbb{X}(x) \rightarrow F$, which can be seen as an element of $V_{F}^{V_{G}}$. We thus obtain a map $\varphi: \Omega \rightarrow V_{F}^{V_{G}}$. By Lemma 5.7 below, $\varphi$ can be chosen to be $\mu$-measurable. Since $x \in \mathbb{X}_{i, j}$ implies $(\varphi(x)(i), \varphi(x)(j)) \in E_{F}$, we have $\varphi\left(\mathbb{X}_{i, j}\right) \subset A_{i, j}(F, G)$ for all $(i, j) \in E_{G}$. Let $m:=\varphi_{\#}(\mu) \in \mathcal{M}^{1}\left(V_{F}^{V_{G}}\right)$. Then $m\left(A_{i, j}(F, G)\right) \geq \mu\left(\mathbb{X}_{i, j}\right)>c(F, G)$. This is absurd by definition of $c(F, G)$.

Reasoning as in Corollary 3.4 we obtain:

Corollary 5.5. Suppose $c(F, G)<1$. If $\inf _{e \in \mathbb{N}^{(2)}} \mu\left(\mathbb{X}_{e}\right) \geq \lambda$, then $\mu(P) \geq$ $\frac{\lambda-c(F, G)}{1-c(F, G)}$.

Remark 5.6. If the sup in the definition of $c(F, G)$ is not reached, it suffices to have the weak inequality $\inf _{e \in E_{G}} \mu\left(\mathbb{X}_{e}\right) \geq c(F, G)$ in order to have $\mu(P)>$ 0 (this is indeed the case of Theorem 4.5). 
It remains to show that the map $\varphi: \Omega \rightarrow V_{F}^{V_{G}}$ in the proof of Theorem 5.4 can be taken to be $\mu$-measurable.

Lemma 5.7. Let $F, G$ be countable directed graphs, let $(\Omega, \mathcal{A}, \mu)$ be a probability space, and let $\mathbb{X}: \Omega \rightarrow 2^{E_{G}}$ be a random subgraph of $G$.

(1) The set $\Omega_{0}:=\{x \in \Omega: \mathbb{X}(x) \rightarrow F\}$ is $\mu$-measurable (i.e. measurable with respect to the $\mu$-completion of $\mathcal{A})$.

(2) There is an $\mu$-measurable function $\varphi: \Omega_{0} \rightarrow V_{F}^{V_{G}}$ that selects, for each $x \in \Omega_{0}$, a graph morphism $\varphi(x): \mathbb{X}(x) \rightarrow F$.

(3) If $F$ is finite, then $\Omega_{0}$ is measurable and $\varphi$ can be chosen measurable.

Proof. Given a function $f: V_{G} \rightarrow V_{F}$, we have $f: \mathbb{X}(x) \rightarrow F$ (i.e., $f$ is a graph morphism from $\mathbb{X}(x)$ to $F)$ if and only if $x \in \bigcap_{(i, j) \in V_{G}} \bigcup_{(a, b) \in V_{F}} B_{i, j, a, b}$, where $x \in B_{i, j, a, b}$ says that $f(i)=a, f(j)=b$ and $x \in \mathbb{X}_{i, j}$. This shows that $B:=\{(x, f): f: \mathbb{X}(x) \rightarrow F\}$ is a measurable subset of $\Omega \times V_{G}^{V_{F}}$. We are looking for a $(\mu-)$ measurable function $\varphi: \pi_{X}(B) \rightarrow V_{F}^{V_{G}}$ whose graph is contained in $B$.

Special case: Let us first assume that $\Omega$ is a Polish space (i.e., a complete separable metric space) with its algebra $\mathcal{A}$ of Borel sets. By Jankov - von Neumann uniformization theorem (see [K:95, Thm. 29.9]), if $X, Y$ are Polish spaces and $Q \subset X \times Y$ is a Borel set, then the projection $\pi_{X}(Q) \subset X$ is universally measurable (i.e. it is $m$-measurable for every $\sigma$-finite Borel measure $m$ on $X)$, and there is a universally measurable function $f: \pi_{X}(Q) \rightarrow Y$ whose graph is contained in $Q$. We can apply this to $X=\Omega, Y=V_{F}^{V_{G}}$ and $Q=B$ to obtain (1) and (2). It remains to show that if $F$ is finite $\pi_{X}(Q)$ and $f$ can be chosen to be Borel measurable. To this aim it suffices to use the following uniformization theorem of Arsenin - Kunugui (see K:95, Thm. 35.46]): if $X, Y, Q$ are as above and each section $Q_{x}=\{y \in Y:(x, y) \in Q\}$ is a countable unions of compact sets, then $p_{X}(Q)$ is Borel and there is a Borel measurable function $f: \pi_{X}(Q) \rightarrow Y$ whose graph is contained in $Q$.

General case: We reduce to the special case as follows. Let $X=2^{V_{G}}, Y=$ $V_{F}^{V^{G}}$ and consider the set $B^{\prime} \subset X \times Y$ consisting of those pairs $(H, f)$ such that $H$ is a subgraph of $G$ (with the same vertices) and $f: H \rightarrow F$ is a graph morphism. Consider the pushforward measure $m=\mathbb{X}_{\#}(\mu)$ defined on the Borel algebra of $2^{V_{G}}$. By the special case there is a $(m$-)measurable function $\psi: \pi_{X}\left(B^{\prime}\right) \rightarrow V_{F}^{V_{G}}$ whose graph is contained in $B^{\prime}$. To conclude it suffices to take $\varphi:=\psi \circ \mathbb{X}$.

We now show how to compute the relative capacity $c\left(F,\left(\mathbb{N}, \mathbb{N}^{(2)}\right)\right.$ ) (see Definition 5.3) for any finite graph $F$. The following invariant of directed graphs has been studied in [R:82] and [FT:85, Section 3].

Definition 5.8. Given a directed graph $F$, we define the capacity of $F$ as

$$
c_{0}(F):=\sup _{\lambda \in \Sigma_{F}} \sum_{(a, b) \in E_{F}} \lambda_{a} \lambda_{b} \in[0,1],
$$

where $\Sigma_{F}$ is the symplex of all sequences $\left\{\lambda_{a}\right\}_{a \in V_{F}}$ of real numbers such that $\lambda_{a} \geq 0$ and $\sum_{a \in V_{F}} \lambda_{a}=1$. 
Proposition 5.9. If $F$ is a finite directed graph, then

$$
c\left(F,\left(\mathbb{N}, \mathbb{N}^{(2)}\right)\right)=c_{0}(F) .
$$

Proof. Let $G=\left(\mathbb{N}, \mathbb{N}^{(2)}\right)$. The proof is a series of reductions.

Step 1. Note that if $\sigma \in \operatorname{Incr}(\mathbb{N})$, then $\sigma \cdot m\left(A_{i, j}(F, G)\right)=m\left(A_{\sigma(i), \sigma(j)}\right)$. Hence the infimum in $(5.2)$ can only increase replacing $m$ with $\sigma_{\#}^{*}(m)$. By Theorem B.8 there is $\sigma \in \operatorname{Incr}(\mathbb{N})$ such that $\sigma \cdot m$ is asymptotically exchangeable. It then follows that we can equivalently take the supremum in (5.2) among the measures $m \in \mathcal{M}^{1}\left(V_{F}^{\mathbb{N}}\right)$ which are asymptotically exchangeable. Step 2. By definition if $m$ is asymptotically exchangeable there is an exchangeable measure $m^{\prime}$ such that $\lim _{k \rightarrow \infty} m_{k}=m^{\prime}$, where $m_{k}=\mathrm{S}^{k} \cdot m$. Clearly

$$
\inf _{(i, j) \in E_{G}} m\left(A_{i, j}(F, G)\right) \leq \lim _{k \rightarrow \infty} m_{k}\left(A_{0,1}(F, G)\right)=m^{\prime}\left(A_{0,1}(F, G)\right) .
$$

So the supremum in (5.2) coincides with $\sup _{m} m\left(A_{0,1}(F, G)\right)$, for $m$ ranging over the exchangeable measures.

Step 3. Recalling $(\overline{\mathrm{B} .13})$, every exchangeable measure is a convex integral combination of Bernoulli measures $B_{\lambda}$, with $\lambda \in \Sigma_{F}$. It follows that it is sufficient to compute the supremum on the Bernoulli measures $B_{\lambda}$. We have:

$$
\begin{aligned}
B_{\lambda}\left(\left\{x \in V_{F}^{\mathbb{N}}:\left(x_{0}, x_{1}\right) \in E_{F}\right\}\right) & =\sum_{(a, b) \in E_{F}} B_{\lambda}\left(\left\{x: x_{0}=a, x_{1}=b\right\}\right) \\
& =\sum_{(a, b) \in E_{F}} \lambda_{a} \lambda_{b}
\end{aligned}
$$

so that $(5.2)$ reduces to $(5.3)$.

Notice that if there is a morphism of graphs from $G$ to $F$, then $c_{0}(G) \leq$ $c_{0}(F)$. Also note that $c_{0}(F)=1$ if there is some $a \in V_{F}$ with $(a, a) \in$ $E_{F}$. Recall that $F$ is said to be: irreflexive if $(a, a) \notin E_{F}$ for all $a \in V_{F}$; symmetric if $(a, b) \in E_{F} \Longleftrightarrow(b, a) \in E_{F}$ for all $a, b \in V_{F}$; anti-symmetric if $(a, b) \in E_{F} \Longrightarrow(b, a) \notin E_{F}$ for all $a, b \in V_{F}$.

The clique number $\operatorname{cl}(F)$ of $F$ is defined as the largest integer $n$ such that there is a subset $S \subset V_{F}$ of size $n$ which forms a clique, namely $(a, b) \in E_{F}$ or $(b, a) \in E_{F}$ for all $a, b \in S$.

Proposition 5.10. (see also FT:85, Section 3]) Let $F$ be a finite irreflexive directed graph. If $F$ is anti-symmetric, then

$$
c_{0}(F)=\frac{1}{2}\left(1-\frac{1}{\mathrm{cl}(F)}\right) .
$$

If $F$ is symmetric, then

$$
c_{0}(F)=1-\frac{1}{\operatorname{cl}(F)} .
$$

In particular $c_{0}\left(K_{p}\right)=1-\frac{1}{p}$.

Proof. The anti-symmetric case follows from the symmetric one taking the symmetric closure. So we can assume that $F$ is symmetric. Let $\lambda \in \Sigma_{F}$ be a maximizing distribution, meaning that $c_{0}(F)=\sum_{(a, b) \in E_{F}} \lambda_{a} \lambda_{b}$, and let $S_{\lambda}$ 
be the subgraph of $F$ spanned by the support of $\lambda$, that is $V_{S_{\lambda}}=\left\{a \in V_{F}\right.$ : $\left.\lambda_{a}>0\right\}$. Given $a \in S_{\lambda}$ note that $\frac{\partial}{\partial \lambda_{a}} \sum_{(u, v) \in E_{F}} \lambda_{u} \lambda_{v}=2 \sum_{b \in V_{F}:(a, b) \in E_{F}} \lambda_{b}$. From Lagrange's multiplier Theorem it then follows that $\sum_{b \in V_{F}}:(a, b) \in E_{F} \lambda_{b}$ is constant, namely it does not depend on the choice of $a \in S_{\lambda}$. Since $\sum_{a \in S_{\lambda}}\left(\sum_{b:(a, b) \in E_{F}} \lambda_{a}\right)=c_{0}(F)$, it follows that for each $a \in S_{\lambda}$ we have:

$$
\sum_{b \in V_{F}:(a, b) \in E_{F}} \lambda_{b}=c_{0}(F) .
$$

If $c, c^{\prime} \in V_{S_{\lambda}}$, we can consider the distribution $\lambda^{\prime} \in \Sigma_{F}$ such that $\lambda_{c}^{\prime}=0$, $\lambda_{c^{\prime}}^{\prime}=\lambda_{c}+\lambda_{c^{\prime}}$, and $\lambda_{b}^{\prime}=\lambda_{b}$ for all $b \in V_{F} \backslash\left\{c, c^{\prime}\right\}$. From (5.7) it then follows that $\lambda^{\prime}$ is also a maximizing distribution whenever $\left(c, c^{\prime}\right) \notin E_{F}$. (In fact $\sum_{(a, b) \in E_{F}} \lambda_{a}^{\prime} \lambda_{b}^{\prime}=\sum_{(a, b) \in E_{F}} \lambda_{a} \lambda_{b}-\lambda_{c} \sum_{b:(c, b) \in E_{F}} \lambda_{b}+\lambda_{c} \sum_{b:\left(c^{\prime}, b\right) \in E_{F}} \lambda b=$ $c_{0}(F)-\lambda_{c} c_{0}(F)+\lambda_{c} c_{0}(F)$.)

As a first consequence, $S_{\lambda}$ is a clique whenever $\lambda$ is a maximizing distribution with minimal support. Indeed, let $K$ be a maximal clique contained in $S_{\lambda}$, and assume by contradiction that there exists $a \in V_{S_{\lambda}} \backslash V_{K}$. Letting $a^{\prime} \in V_{K}$ be a vertex of $F$ independent of $a$ (such an element exists since $K$ is a maximal clique), and letting $\lambda^{\prime} \in \Sigma_{F}$ as above, we have $c_{0}(F)=\sum_{(a, b) \in E_{F}} \lambda_{a}^{\prime} \lambda_{b}^{\prime}$, contradicting the minimality of $V_{S_{\lambda}}$.

Once we know that $S_{\lambda}$ is a clique, again from (5.7) we get that $\lambda$ is a uniform ditribution, that is $\lambda_{a}=\lambda_{b}$, for all $a, b \in V_{S_{\lambda}}$. It follows

$$
c_{0}(F)=1-\frac{1}{\left|S_{\lambda}\right|} \leq 1-\frac{1}{\operatorname{cl}(F)},
$$

which in turn implies (5.5), the opposite inequality being realized by a uniform distribution on a maximal clique.

Notice that the proof of Proposition 5.10 shows that there exists a maximizing $\lambda \in \Sigma_{F}$ whose support is a clique (not necessarily of maximal order).

5.1. Chromatic number. We will apply the results of the previous section to study the chromatic number of a random subgraph of $\left(\mathbb{N}, \mathbb{N}^{(2)}\right)$. We point out that an alternative proof of this result follows from [EH:64, Theorem 1].

We recall that the chromatic number $\chi(G)$ of a directed graph $G$ is the smallest $n$ such that there is a colouring of the vertices of $G$ with $n$ colours in such a way that $a, b \in V_{G}$ have different colours whenever $(a, b) \in E_{G}$ (see (B:79).

For $p \in \mathbb{N}$, let $K_{p}$ be the complete graph on $p$ vertices, namely $K_{p}$ has set of vertices $p=\{0,1, \ldots, p-1\}$ and set of edges $\left\{(x, y) \in p^{2}: x \neq y\right\}$. Clearly $\chi\left(K_{p}\right)=p$. Note also that:

$$
G \rightarrow K_{p} \Longleftrightarrow \chi(G) \leq p .
$$

Now let $(\Omega, \mathcal{A}, m)$ be a probability space, and let $\mathbb{X}: \Omega \rightarrow 2^{E_{G}}$ be a random subgraph of $G=\left(\mathbb{N}, \mathbb{N}^{(2)}\right)$. Let $P=\{x \in \Omega: \chi(\mathbb{X}(x)) \geq p\}$. By Equation (5.8) and the results of the previous section, if $\inf _{e \in \mu\left(\mathbb{X}_{e}\right)}>c\left(K_{p},\left(\mathbb{N}, \mathbb{N}^{(2)}\right)\right.$, then $\mu(P)>0$. This however does not say much unless we manage to determine $c\left(K_{p},\left(\mathbb{N}, \mathbb{N}^{(2)}\right)\right)$. We will show that $c\left(K_{p},\left(\mathbb{N}, \mathbb{N}^{(2)}\right)\right)=\left(1-\frac{1}{p}\right)$, so we have: 
Theorem 5.11. Let $(\Omega, \mathcal{A}, m)$ be a probability space, and let $\mathbb{X}: \Omega \rightarrow 2^{E_{G}}$ be a random subgraph of $\left(\mathbb{N}, \mathbb{N}^{(2)}\right)$. If $\inf _{e \in \mu\left(\mathbb{X}_{e}\right)}>1-\frac{1}{p}$, then

$$
\mu(\{x \in \Omega: \chi(\mathbb{X}(x)) \geq p+1\})>0 .
$$

\section{INFINITE CLIQUES}

We recall the following standard Borel-Cantelli type result, which shows that Problem 3 has a positive answer for $k=1$.

Proposition 6.1. Let $(\Omega, \mathcal{A}, \mu)$ be a probability space. Let $\lambda>0$ and for each $i \in \mathbb{N}$ let $X_{i} \subseteq \Omega$ be a measurable set such that $\mu\left(X_{i}\right) \geq \lambda$. Then there is an infinite set $J \subset \mathbb{N}$ such that

$$
\bigcap_{i \in J} X_{i} \neq \emptyset
$$

Proof. The set $Y:=\bigcap_{n} \bigcup_{i>n} X_{i}$ is a decreasing intersection of sets of (finite) measure greater than $\lambda>0$, hence $\mu(Y) \geq \lambda$ and, in particular, $Y$ is nonempty. Now it suffices to note that any element $x$ of $Y$ belongs to infinitely many $X_{i}$ 's.

Proposition 6.1 has the following interpretation: if we choose each element of $\mathbb{N}$ with probability greater or equal to $\lambda$, we obtain an infinite subset with probability greater or equal to $\lambda$.

The following example shows that Problem 3 has in general a negative answer for $k>1$.

Example 6.2. Let $p \in \mathbb{N}$ and consider the Cantor space $\Omega=p^{\mathbb{N}}$, equipped with the Bernoulli measure $B_{(1 / p, \ldots, 1 / p)}$, and let $\mathbb{X}_{i, j}:=\left\{x \in \Omega: x_{i} \neq x_{j}\right\}$. Then each $\mathbb{X}_{i, j}$ has measure $\lambda=1-1 / p$, and for all $x \in X$ the graph $\mathbb{X}(x):=\left\{(i, j) \in \mathbb{N}^{(2)}: x \in \mathbb{X}_{i, j}\right\}$ does not contains cliques (i.e. complete subgraphs) of cardinality $(p+1)$.

In view of Example 6.2, we need further assumptions in order to get a positive answer to Problem 3 .

Example 6.3. By Ramsey theorem, Problem 3 has a positive answer if there is a finite set $S \subset \Omega$ such that each $X_{i_{1}, \ldots, i_{k}}$ has a non-empty intersection with $S$. In particular, this is the case if $\Omega$ is countable.

Proposition 6.4. Let $r>0$. Assume that $\Omega$ is a compact metric space and each set $\mathbb{X}_{i_{1}, \ldots, i_{k}}$ contains a ball $B_{i_{1}, \ldots, i_{k}}$ of radius $r>0$. Then Problem 3 has a positive answer.

Proof. Applying Lemma A.1 to the centers of the balls $B_{i_{1}, \ldots, i_{k}}$ it follows that for all $0<r^{\prime}<r$ there exists an infinite set $J$ and a ball $B$ of radius $r^{\prime}$ such that

$$
B \subset \bigcap_{\left(j_{1}, \ldots, j_{k}\right) \in J^{[k]}} X_{j_{1}, \ldots, j_{k}}
$$

We now give a sufficient condition for a positive answer to Problem 3 . 
Theorem 6.5. Let $(\Omega, \mu)$ be a probability space. Let $\lambda>0$ and assume that we have the sets $\mu\left(\mathbb{X}_{i_{1} \ldots i_{k}}\right) \geq \lambda$ for each $\left(i_{1}, \ldots, i_{k}\right) \in \mathbb{N}^{(k)}$. Assume further that the indicator functions of $\mathbb{X}_{i_{1}, \ldots, i_{k}}$ belong to a compact subset $\mathcal{K}$ of $L^{1}(\Omega, \mu)$. Then, for any $\varepsilon>0$ there exists an infinite set $J \subset \mathbb{N}$ such that

$$
\mu\left(\bigcap_{\left(i_{1}, \ldots, i_{k}\right) \in J^{[k]}} X_{i_{1} \ldots i_{k}}\right) \geq \lambda-\varepsilon
$$

Proof. Consider first the case $k=1$. By compactness of $\mathcal{K}$, for all $\varepsilon>0$ there exist an increasing sequence $\left\{i_{n}\right\}$ and a set $X_{\infty} \subset X$, with $\mu\left(X_{\infty}\right) \geq \lambda$, such that

$$
\mu\left(X_{\infty} \Delta X_{i_{n}}\right) \leq \frac{\varepsilon}{2^{n}} \quad \forall n \in \mathbb{N} .
$$

As a consequence, letting $J:=\left\{i_{n}: n \in \mathbb{N}\right\}$ we have

$$
\mu\left(\bigcap_{n \in \mathbb{N}} X_{i_{n}}\right) \geq \mu\left(X_{\infty} \cap \bigcap_{n \in \mathbb{N}} X_{i_{n}}\right) \geq \mu\left(X_{\infty}\right)-\sum_{n \in \mathbb{N}} \mu\left(X_{\infty} \Delta X_{i_{n}}\right) \geq \lambda-\varepsilon .
$$

For $k>1$, we apply Lemma A.1 with

$$
\begin{aligned}
M & =\mathcal{K} \subset L^{1}(\Omega, \mu) \\
f\left(i_{1}, \ldots, i_{k}\right) & =\chi_{X_{i_{1} \ldots i_{k}}} \in L^{1}(\Omega, \mu) .
\end{aligned}
$$

In particular, recalling Remark A.4, for all $\varepsilon>0$ there exist $J=\sigma(\mathbb{N})$, $X_{\infty} \subset \Omega$, and $X_{i_{1} \ldots i_{m}} \subset X$, for all $\left(i_{1}, \ldots, i_{m}\right) \in J^{[m]}$ with $1 \leq m<k$, such that $\mu\left(X_{\infty}\right) \geq \lambda$ and for all $\left(i_{1}, \ldots, i_{k}\right) \in J^{[k]}$ it holds

$$
\begin{aligned}
\mu\left(X_{\infty} \Delta X_{i_{1}}\right) & \leq \frac{\varepsilon}{2^{\sigma^{-1}\left(i_{1}\right)}} \\
\mu\left(X_{i_{1} \ldots i_{m}} \Delta X_{i_{1} \ldots i_{m+1}}\right) & \leq \frac{\varepsilon}{2^{\sigma^{-1}\left(i_{m+1}\right)}} .
\end{aligned}
$$

Reasoning as above, it then follows

$$
\begin{aligned}
& \mu\left(X_{\infty} \Delta \bigcap_{\left(i_{1}, \ldots, i_{k}\right) \in J^{[k]}} X_{i_{1} \ldots i_{k}}\right) \leq \\
& \sum_{i_{1} \in \mathbb{N}} \mu\left(X_{\infty} \Delta X_{i_{1}}\right)+\sum_{i_{1}<i_{2}} \mu\left(X_{i_{1}} \Delta X_{i_{1} i_{2}}\right)+ \\
& \cdots+\sum_{i_{1}<\cdots<i_{k}} \mu\left(X_{i_{1} \ldots i_{k-1}} \Delta X_{i_{1} \ldots i_{k}}\right) \leq C(k) \varepsilon,
\end{aligned}
$$

where $C(k)>0$ is a constant depending only on $k$. Therefore

$$
\begin{aligned}
\mu\left(\bigcap_{\left(i_{1}, \ldots, i_{k}\right) \in J^{[k]}} X_{i_{1} \ldots i_{k}}\right) & \geq \mu\left(X_{\infty} \cap \bigcap_{\left(i_{1}, \ldots, i_{k}\right) \in J^{[k]}} X_{i_{1} \ldots i_{k}}\right) \\
& \geq \mu\left(X_{\infty}\right)-\mu\left(X_{\infty} \Delta \bigcap_{\left(i_{1}, \ldots, i_{k}\right) \in J^{[k]}} X_{i_{1} \ldots i_{k}}\right) \\
& \geq \lambda-C(k) \varepsilon .
\end{aligned}
$$


Notice that from Theorem 6.5 it follows that Problem 3 has a positive answer if there exist an infinite $J \subseteq \mathbb{N}$ and sets $\widetilde{\mathbb{X}}_{i_{1}, \ldots, i_{k}} \subseteq X_{i_{1} \ldots i_{k}}$ with $\left(i_{1}, \ldots, i_{k}\right) \in J^{[k]}$, such that $\mu\left(\widetilde{\mathbb{X}}_{i_{1}, \ldots, i_{k}}\right) \geq \lambda$ for some $\lambda>0$, and the indicator functions of $\widetilde{\mathbb{X}}_{i_{1}, \ldots, i_{k}}$ belong to a compact subset of $L^{1}(\Omega, \mu)$.

Remark 6.6. We recall that, when $\Omega$ is a compact subset of $\mathbb{R}^{n}$ and the perimeters of the sets $\mathbb{X}_{i_{1}, \ldots, i_{k}}$ are uniformly bounded, then the family $\chi_{\mathbb{X}_{i_{1}, \ldots, i_{k}}}$ has compact closure in $L^{1}(\Omega, \mu)$ (see for instance AFP:00, Thm. 3.23]). In particular, if the sets $\mathbb{X}_{i_{1}, \ldots, i_{k}}$ have equibounded Cheeger constant, i.e. if there exists $C>0$ such that

$$
\min _{E \subset \mathbb{X}_{i_{1}, \ldots, i_{k}}} \frac{\operatorname{Per}(E)}{|E|} \leq C \quad \forall\left(i_{1}, \ldots, i_{k}\right) \in \mathbb{N}^{(k)},
$$

then Problem 3 has a positive answer.

\section{Appendix A. A topological Ramsey theorem}

The following metric version of Ramsey theorem reduces to the classical Ramsey theorem when $M$ is finite.

Lemma A.1. Let $M$ be a compact metric space, let $k \in \mathbb{N}$, and let $f$ : $\mathbb{N}^{(k)} \rightarrow M$. Then there exists an infinite set $J \subset \mathbb{N}$ such that the limit

$$
\lim _{\substack{\left(i_{1}, \ldots, i_{k}\right) \rightarrow+\infty \\\left(i_{1}, \ldots, i_{k}\right) \in J^{(k)}}} f\left(i_{1}, \ldots, i_{k}\right)
$$

exists.

Proof. Notice first that the thesis is trivial for $k=1$, since the space $M$ is compact. Assuming that the thesis holds for some $k \in \mathbb{N}$, we want to prove it for $k+1$. So let $f: \mathbb{N}^{(k+1)} \rightarrow M$. By inductive assumption, for all $j \in \mathbb{N}$ there exist a infinite set $J_{j} \subset \mathbb{N}$ and a point $x_{j} \in M$ such that $x_{j}=$ $\lim _{i_{1}, \ldots, i_{k} \rightarrow \infty} f\left(j, i_{1}, \ldots, i_{k}\right)$, with $\left(i_{1}, \ldots, i_{k}\right) \in\left[J_{j}\right]^{k}$. Possibly extracting further subsequences we can also assume that

$$
d\left(x_{j}, f\left(j, i_{1}, \ldots, i_{k}\right)\right) \leq 1 / 2^{j}
$$

for all $\left(i_{1}, \ldots, i_{k}\right) \in J_{j}^{(k)}$. Moreover, by a recursive construction, we can assume that $J_{j+1} \subseteq J_{j}$. Now define $\tau \in \operatorname{Incr}(\mathbb{N})$ by choosing $\tau(0) \in \mathbb{N}$ and inductively $\tau(n+1) \in J_{\tau(n)}$. Since $J_{j+1} \subset J_{j}$ for all $j$, this implies $\tau(m) \in J_{\tau(n)}$ for all $m>n$. By compactness of $M$, there exists $\lambda \in \operatorname{Incr}(\mathbb{N})$ and a point $x \in M$ such that $x_{\tau(\lambda(n))} \rightarrow x$ for $n \rightarrow \infty$. Take $J=\operatorname{Im}(\tau \circ \lambda)$. The thesis follows the triangle inequality $d\left(x, f\left(j, i_{1}, \ldots, i_{k}\right)\right) \leq d\left(x, x_{j}\right)+$ $d\left(x_{j}, f\left(j, i_{1}, \ldots, i_{k}\right)\right)$, noting that if $j<i_{1}<\ldots<i_{k}$ are in $J$, then $i_{1}, \ldots, i_{k} \in J_{j}$ (so Equation A.1 applies).

Note that in Lemma A.1, the condition $\left(i_{1}, \ldots, i_{k}\right) \rightarrow+\infty$ is equivalent to $i_{1} \rightarrow \infty$ (since $i_{1}<i_{2}<\ldots<i_{k}$ ). We would like to strengthen Lemma A.1 by requiring the existence of all the partial limits

$$
x=\lim _{i_{j(1)} \rightarrow \infty} \lim _{i_{j(2)} \rightarrow \infty} \cdots \lim _{i_{j(r)} \rightarrow \infty} x_{i_{1} \ldots i_{k}}
$$


where $1 \leq r \leq k$ and $\left(i_{j(1)}, \ldots, i_{j(r)}\right) \in J^{(r)}$ is a subsequence of $\left(i_{1}, \ldots, i_{k}\right) \in$ $J^{(k)}$. Note that the existence of all these $2^{k-1}$ partial limits does not follow from Lemma A.1. For instance $\lim _{(i, j) \rightarrow \infty} \frac{(-1)^{j}}{i+1}=0$ but $\lim _{i \rightarrow \infty} \lim _{j \rightarrow \infty} \frac{(-1)^{j}}{i+1}$ does not exist.

To prove the desired strengthening it is convenient to introduce some terminology. Let $\overline{\mathbb{N}}=\mathbb{N} \cup\{\infty\}$ be the one-point compactification of $\mathbb{N}$. Given a distance $\delta$ on $\mathbb{N}$, we consider on $\mathbb{N}^{(k)}$ the induced metric

$$
\delta_{k}\left(\left(n_{1}, \ldots, n_{k}\right),\left(m_{1}, \ldots, m_{k}\right)\right):=\max _{i} \delta\left(n_{i}, m_{i}\right) .
$$

Given $\sigma \in \operatorname{Incr}(\mathbb{N})$, let $\sigma_{*}: \mathbb{N}^{(k)} \rightarrow \mathbb{N}^{(k)}$ be the induced map defined by $\sigma_{*}\left(n_{1}, \ldots, n_{k}\right):=\left(\sigma\left(n_{1}\right), \ldots, \sigma\left(n_{k}\right)\right)$. Given $f: \mathbb{N}^{(k)}$, by the following theorem there is an infinite $J \subset \mathbb{N}$ such that all the partial limits of $f\left\lceil_{J^{(k)}}\right.$ exist. Moreover the arbitrarity of $\delta$ shows that we can impose an arbitrary modulus of convergence on all the partial limits of $f \circ \sigma_{*}$, where $\sigma \in \operatorname{Incr}(\mathbb{N})$ is an increasing enumeration of $J$.

Theorem A.2. Let $M$ be a compact metric space, let $k \in \mathbb{N}$, and let $f$ : $\mathbb{N}^{(k)} \rightarrow M$. Then, for any distance $\delta$ on $\overline{\mathbb{N}}$ there exists $\sigma \in \operatorname{Incr}(\mathbb{N})$ such that $f \circ \sigma_{*}: \mathbb{N}^{(k)} \rightarrow M$ is 1-Lipschitz. As a consequence, it can be extended to a 1-Lipschitz function on the closure of $\mathbb{N}^{(k)}$ in $\overline{\mathbb{N}}^{k}$.

Lemma A.3. Let $\delta$ be a metric on $\overline{\mathbb{N}}$. Then there is another metric $\delta^{*}$ on $\overline{\mathbb{N}}$ such that

(1) $\delta^{*}(x, y) \leq \delta(x, y)$ for all $x, y$.

(2) $\delta^{*}$ is monotone in the following sense: $\delta^{*}\left(x^{\prime}, y^{\prime}\right) \leq \delta^{*}(x, y)$ for all $x, x, y, y^{\prime}$, provided $x<\min \left(y, x^{\prime}, y^{\prime}\right)$.

(3) $\varepsilon^{*}(x) \geq \varepsilon^{*}(y)$ for all $x \leq y$, where

$$
\varepsilon^{*}(x):=\min _{y \geq x+1} \delta^{*}(x, y) .
$$

Proof. We shall define a distance of the form $\delta^{*}(x, y)=\delta(\psi(x), \psi(y))$ for a suitable strictly increasing function

$$
\psi: \overline{\mathbb{N}} \rightarrow \overline{\mathbb{N}} .
$$

To this aim, let us consider, for any $x \in \overline{\mathbb{N}}$, the diameter of the interval $[x, \infty] \cap \overline{\mathbb{N}}$

$$
\eta(x):=\max _{x \leq y \leq z} \delta(y, z)
$$

and the point-set distance from $x$ to the interval $[x+1, \infty] \cap \overline{\mathbb{N}}$

$$
\varepsilon(x):=\min _{y \geq x+1} \delta(x, y) .
$$

Since $\varepsilon(x)>0$ for all $x<\infty$ and $\eta(x)=o(1)$ as $x \rightarrow \infty$, there exists a recursively defined, strictly increasing function $\psi: \overline{\mathbb{N}} \rightarrow \overline{\mathbb{N}}$ such that for any $x \in \mathbb{N}$

$$
\begin{aligned}
\eta(\psi(x)) & \leq \varepsilon(x) \\
\eta(\psi(x+1)) & \leq \varepsilon(\psi(x)) .
\end{aligned}
$$


As a consequence, the distance

$$
\delta^{*}(x, y):=\delta(\psi(x), \psi(y))
$$

verifies, for all $x<y \leq \infty$

$$
\delta^{*}(x, y)=\delta(\psi(x), \psi(y)) \leq \eta(\psi(x)) \leq \varepsilon(x) \leq \delta(x, y),
$$

and, assuming also $x<x^{\prime} \leq \infty$ and $x<y^{\prime} \leq \infty$,

$$
\begin{aligned}
\delta^{*}\left(x^{\prime}, y^{\prime}\right) & =\delta\left(\psi\left(x^{\prime}\right), \psi\left(y^{\prime}\right)\right) \leq \eta\left(\psi\left(x^{\prime}\right)\right) \leq \eta(\psi(x+1)) \\
& \leq \varepsilon(\psi(x)) \leq \delta(\psi(x), \psi(y))=\delta^{*}(x, y) .
\end{aligned}
$$

To prove the last statement we observe that

$$
\varepsilon^{*}(x) \geq \varepsilon(\psi(x)) \geq \eta(\psi(x+1)) \geq \varepsilon^{*}(x+1) .
$$

Proof of Theorem A.2. By Lemma A.3 we can assume that $\delta$ is monotone in the sense of Lemma A.3 (2).

We proceed by induction on $k$. When $k=1$, consider the function $\varepsilon(n):=$ $\min _{m \geq n+1} \delta(n, m)$ as in (A.2). By compactness of $M$ there exist $x \in M$ and a subsequence $f \circ \sigma$ of $f$ converging to $x$ with the property

$$
d_{M}(f(\sigma n), x) \leq \frac{\varepsilon(n)}{2} .
$$

Recalling Lemma A.3 (3), for $n \neq m$ we have

$$
d_{M}(f(\sigma n), f(\sigma m)) \leq \frac{\varepsilon(n)+\varepsilon(m)}{2} \leq \delta(n, m) .
$$

So $f \circ \sigma$ is 1-Lipschitz.

Now assume inductively that the thesis holds for some $k \in \mathbb{N}$, and let us prove it for $k+1$. So let $f: \mathbb{N}^{(k+1)} \rightarrow M$. We need to prove the existence of $\sigma \in \operatorname{Incr}(\mathbb{N})$ such that

$$
d_{M}\left(f\left(\sigma_{*}(n, \boldsymbol{m})\right), f\left(\sigma_{*}\left(n^{\prime}, \boldsymbol{m}^{\prime}\right)\right)\right) \leq \delta_{k+1}\left((n, \boldsymbol{m}),\left(n^{\prime}, \boldsymbol{m}^{\prime}\right)\right)
$$

for all $(n, \boldsymbol{m}) \in \mathbb{N}^{(k+1)}$ and $\left(n^{\prime}, \boldsymbol{m}^{\prime}\right) \in \mathbb{N}^{(k+1)}$, where $\boldsymbol{m}=\left(m_{1}, \ldots, m_{k}\right)$ and $\boldsymbol{m}^{\prime}=\left(m_{1}^{\prime}, \ldots, m_{k}^{\prime}\right)$.

Given $n \in \mathbb{N}$ define $f_{n}: \mathbb{N}^{(k)} \rightarrow M$ by

$$
f_{n}(\boldsymbol{m}):= \begin{cases}f(n, \boldsymbol{m}) & \text { if } n<m_{1}, \\ \perp & \text { if } n \geq m_{1}\end{cases}
$$

where $\perp$ is an arbitrary element of $M$. Note that the condition $n<m_{1}$ is equivalent to $(n, \boldsymbol{m}) \in \mathbb{N}^{(k+1)}$.

By inductive assumption, for all $n \in \mathbb{N}$ there exists $\theta_{n} \in \operatorname{Incr}(\mathbb{N})$ such that $f_{n} \circ \theta_{n *}: \mathbb{N}^{(k)} \rightarrow M$ is 1-Lipschitz. By a recursive construction, we can also assume that $\theta_{n+1}$ is a subsequence of $\theta_{n}$, namely $\theta_{n+1}=\theta_{n} \circ \gamma_{n}$ for some $\gamma_{n} \in \operatorname{Incr}(\mathbb{N})$. Indeed to obtain $\theta_{n+1}$ as desired it suffices to apply the induction hypothesis to $f_{n+1} \circ \theta_{n *}: \mathbb{N}^{(k)} \rightarrow M$ rather than directly to $f_{n+1}$.

Since $f_{n} \circ \theta_{n *}$ is 1-Lipschitz, there exist the limit

$$
g(n):=\lim _{\min (\boldsymbol{m}) \rightarrow \infty} f\left(n, \theta_{n *}(\boldsymbol{m})\right)
$$


Passing to a subsequence we can further assume that all the values of $f_{n} \circ \theta_{n}$ are within distance $\varepsilon(n) / 4$ from its limit, namely:

$$
d_{M}\left(g(n), f\left(n, \theta_{n}((\boldsymbol{m}))\right)<\frac{\varepsilon(n)}{4} .\right.
$$

Let $J_{n}:=\theta_{n}(\mathbb{N}) \subset \mathbb{N}$ and let $\tau \in \operatorname{Incr}(\mathbb{N})$ be such that:

$$
\tau(n+1) \in J_{\tau(n)}
$$

It then follows that

$$
\forall n, m \in \tau(\mathbb{N}) \quad m>n \Longrightarrow m \in J_{n} .
$$

For later purposes we need to define $\tau(n+1)$ as an element of $J_{\tau(n)}$ bigger than its $n+1$-th element, namely $\tau(n+1)>\theta_{\tau(n)}(n+1)$. So, for the sake of concreteness, we define inductively $\tau(0):=0$ and $\tau(n+1):=\theta_{\tau(n)}(n+2)$. It then follows that:

$$
\forall i, j \in \tau(\mathbb{N}) \forall k \in \mathbb{N} \quad j>i, j \geq k \Longrightarrow \tau(j)>\theta_{\tau(i)}(k) .
$$

Reasoning as in the case $k=1$, there is $\lambda \in \operatorname{Incr}(\mathbb{N})$ and $x_{\infty} \in M$ such that

$$
d_{M}\left(g(\tau(\lambda(n))), x_{\infty}\right)<\frac{\varepsilon(n)}{4}
$$

Now define $\sigma:=\tau \circ \lambda \in \operatorname{Incr}(\mathbb{N})$. Note that $\sigma(\mathbb{N}) \subset \tau(\mathbb{N})$ so $($ A.12 and (A.13) continue to hold with $\sigma$ instead of $\tau$. We claim that $f \circ \sigma_{*}: \mathbb{N}^{(k+1)} \rightarrow$ $M$ is 1-Lipschitz.

As a first step we show that

$$
\exists \boldsymbol{k}>\boldsymbol{m}:\left(f \circ \sigma_{*}\right)(n, \boldsymbol{m})=\left(f_{\sigma(n)} \circ \theta_{\sigma(n)}\right)(n, \boldsymbol{k})
$$

where $\boldsymbol{k}>\boldsymbol{m}$ means that $k_{i}>m_{i}$ for all respective components. To prove (A.15) recall that $\left(f \circ \sigma_{*}\right)(n, \boldsymbol{m})=f\left(\sigma(n), \sigma\left(m_{1}\right), \ldots, \sigma\left(m_{k}\right)\right)$. Since $n<$ $\min (\boldsymbol{m})$, by (A.12) the elements $\sigma\left(m_{1}\right), \ldots, \sigma\left(m_{k}\right)$ are in the image of $\theta_{\sigma(n)}$, namely for each $i$ we have $\sigma\left(m_{i}\right)=\theta_{\sigma(n)}\left(k_{i}\right)$ for some $k_{i} \in \mathbb{N}$. Moreover applying (A.13) we must have $k_{i}>m_{i}$. The proof of (A.15) is thus complete.

It follows from (A.15) and (A.10) that $\left(f \circ \sigma_{*}\right)(n, \boldsymbol{m})$ is within distance $\varepsilon(\sigma(n)) / 4$ from its limit $g(\sigma(n))$, which in turn is within distance $\varepsilon(n) / 4$ from its limit $x_{\infty}$ by (A.14). We thus proved:

$$
d_{M}\left(f\left(\sigma_{*}(n, \boldsymbol{m})\right), x_{\infty}\right)<\frac{1}{4} \varepsilon(\sigma(n))+\frac{1}{4} \varepsilon(n) .
$$

Recalling that for $x \neq y$ we have $\varepsilon(x)+\varepsilon(y) \leq 2 \delta(x, y)$, it follows that for $n \neq n^{\prime}$ the left-hand side of (A.8) is bounded by $\left[\delta\left(\sigma(n), \sigma\left(n^{\prime}\right)\right)+\delta\left(n, n^{\prime}\right)\right] / 2$, which in turn is $\leq \delta\left(n, n^{\prime}\right)$ by monotonicity of $\delta$.

If remains to prove (A.8) in the case $n=n^{\prime}$. Given $\boldsymbol{m}, \boldsymbol{m}^{\prime}$ as in (A.8), we apply (A.15) to get $\boldsymbol{k}>\boldsymbol{m}, \boldsymbol{k}^{\prime}>\boldsymbol{m}^{\prime}$ with $\left(f \circ \sigma_{*}\right)(n, \boldsymbol{m})=\left(f_{\sigma(n)} \circ \theta_{\sigma(n)}\right)(n, \boldsymbol{k})$ and $\left(f \circ \sigma_{*}\right)\left(n, \boldsymbol{m}^{\prime}\right)=\left(f_{\sigma(n)} \circ \theta_{\sigma(n)}\right)\left(n, \boldsymbol{k}^{\prime}\right)$.

Using the monotonicity of $\delta$ and the fact that $f_{\sigma(n)} \circ \theta_{\sigma(n)}$ is 1-Lipschitz, it follows that:

$$
d_{M}\left(f\left(\sigma_{*}(n, \boldsymbol{m})\right), f\left(\sigma_{*}\left(n, \boldsymbol{m}^{\prime}\right)\right)\right) \leq \delta_{k}\left(\boldsymbol{k}, \boldsymbol{k}^{\prime}\right) \leq \delta_{k}\left(\boldsymbol{m}, \boldsymbol{m}^{\prime}\right) .
$$


Remark A.4. Theorem A.2 implies that there exists an infinite set $J=$ $\sigma(\mathbb{N}) \subset \mathbb{N}$ such that, for all $0 \leq m<k$ and $\left(i_{1}, \ldots, i_{m}\right) \in J^{[m]}$, there are limit points $x_{i_{1} \ldots i_{m}} \in M$ with the property

$$
x_{i_{1} \ldots i_{m}}=\lim _{\substack{\left(i_{m+1}, \ldots, i_{k}\right) \rightarrow \infty \\\left(i_{1} \ldots i_{k}\right) \in J\left[J^{[k]}\right.}} x_{i_{1} \ldots i_{k}},
$$

where we set $x_{i_{1} \ldots i_{k}}:=f\left(i_{1}, \ldots, i_{k}\right)$. Moreover, by choosing the distance $\delta(n, m)=\varepsilon\left|2^{-n}-2^{-m}\right|$, we may also require

$$
d_{M}\left(x_{i_{1} \ldots i_{m}}, x_{i_{1} \ldots i_{k}}\right) \leq \frac{\varepsilon}{2^{\sigma^{-1}\left(i_{m+1}\right)}} \quad \forall\left(i_{1}, \ldots, i_{k}\right) \in J^{[k]} .
$$

\section{Appendix B. Exchangeable measures}

Let $\Lambda$ be a compact metric space. We recall a classical notion of exchangeable measure due to De Finetti [DF:74], showing some equivalent conditions.

Proposition B.1. Given $m \in \mathcal{M}^{1}\left(\Lambda^{\mathbb{N}}\right)$, the following conditions are equivalent:

a) $m$ is $\mathfrak{S}_{c}(\mathbb{N})$-invariant;

b) $m$ is $\operatorname{Inj}(\mathbb{N})$-invariant;

c) $m$ is $\operatorname{Incr}(\mathbb{N})$-invariant.

Definition B.2. If $m$ satisfies one of these equivalent conditions we say that $m$ is exchangeable.

Notice that an exchangeable measure is always shift-invariant, while there are shift-invariant measures which are not exchangeable. To prove Proposition B.1 we need some preliminary results concerning measures satisfying condition (c).

Definition B.3. Given $m \in \mathcal{M}\left(\Lambda^{\mathbb{N}}\right)$ and $f \in L^{p}\left(\Lambda^{\mathbb{N}}\right)$, with $p \in[1,+\infty]$, we let

$$
\tilde{f}=E\left(f \mid \mathcal{A}_{s}\right) \in L^{p}\left(\Lambda^{\mathbb{N}}\right)
$$

be the conditional probability of $f$ with respect to the $\sigma$-algebra $\mathcal{A}_{s}$ of the shift-invariant Borel subsets of $\Lambda^{\mathbb{N}}$. In particular, $\tilde{f}$ is shift-invariant, and by Birkhoff's theorem (see for instance $[\mathrm{P}: 82]$ ) we have

$$
\tilde{f}=\lim _{n \rightarrow \infty} \frac{1}{n} \sum_{k=0}^{n-1} f \circ \mathrm{S}^{* k},
$$

where the limit holds almost everywhere and in the strong topology of $L^{1}\left(\Lambda^{\mathbb{N}}\right)$.

Lemma B.4. Assume that $m \in \mathcal{M}^{1}\left(\Lambda^{\mathbb{N}}\right)$ is $\operatorname{Incr}(\mathbb{N})$-invariant. Then for all $f \in L^{\infty}\left(\Lambda^{\mathbb{N}}, m\right)$ we have

$$
\tilde{f}=\lim _{n \rightarrow \infty} f \circ \mathrm{S}^{* n},
$$

where the limit is taken in the weak $k^{*}$ topology of $L^{\infty}\left(\Lambda^{\mathbb{N}}\right)$, namely for every $g \in L^{1}\left(\Lambda^{\mathbb{N}}, m\right)$ we have

$$
\lim _{n \rightarrow \infty} \int_{\Lambda^{\mathbb{N}}} g\left(f \circ \mathbf{S}^{* n}\right) d m=\int_{\Lambda^{\mathbb{N}}} g \tilde{f} d m
$$


Proof. It suffices to prove that $\lim _{n \rightarrow \infty} f \circ \mathrm{S}^{* n}$ exists, since in that case it is necessarily equal to the ( weak $^{*}$ ) limit of the arithmetic means $\frac{1}{n} \sum_{k=0}^{n-1} f \circ \mathrm{S}^{* k}$, and therefore to $\tilde{f}$ (since $\tilde{f}=\lim _{n \rightarrow \infty} \frac{1}{n} \sum_{k=0}^{n-1} f \circ \mathrm{S}^{* k}$ in an even stronger topology). Since the sequence $f \circ \mathrm{S}^{* n}$ is equibounded in $L^{\infty}\left(\Lambda^{\mathbb{N}}, m\right)$, it is enough to prove $(\overline{B .2})$ for all $g$ in a dense subset $D$ of $L^{1}\left(\Lambda^{\mathbb{N}}\right)$. We can take $D$ to be the set of those functions $g \in L^{1}\left(\Lambda^{\mathbb{N}}, m\right)$ depending on finitely many coordinates (namely $g(x)=h\left(x_{1}, \ldots, x_{r}\right)$ for some $r \in \mathbb{N}$ and some $\left.h \in L^{1}\left(\Lambda^{r}, m\right)\right)$. The convergence of (B.2) for $g(x)=h\left(x_{1}, \ldots, x_{r}\right)$ follows at once from the fact that $\sigma \cdot m=m$ for all $\sigma \in \operatorname{Incr}(\mathbb{N})$, which implies that the quantity in $(\overline{\mathrm{B} .2})$ is constant for all $n>r$. Indeed to prove that $\int_{\Lambda^{\mathbb{N}}} g\left(f \circ \mathrm{S}^{* n}\right) d m=\int_{\Lambda^{\mathbb{N}}} g\left(f \circ \mathrm{S}^{* n+l}\right) d m$ it suffices to consider the function $\sigma \in \operatorname{Incr}(\mathbb{N})$ which fixes $0, \ldots, r-1$ and sends $i$ to $i+l$ for $i \geq r$.

We are now ready to prove the equivalence of the conditions in the definition of exchangeable measure.

Proof of Proposition B.1. Since $\mathfrak{S}_{c}(\mathbb{N}) \subset \operatorname{Inj}(\mathbb{N})$ and $\operatorname{Incr}(\mathbb{N}) \subset \operatorname{Inj}(\mathbb{N})$, the implications $b) \Rightarrow$ a) and b) $\Rightarrow$ c) are obvious. The implication a) $\Rightarrow b$ ) is also obvious since it is true on the Borel subsets of $\Lambda^{\mathbb{N}}$ of the form $\left\{x \in \Lambda^{\mathbb{N}}\right.$ : $\left.x_{i_{1}} \in A_{1}, \ldots, x_{i_{r}} \in A_{r}\right\}$, which generate the whole Borel $\sigma$-algebra of $\Lambda^{\mathbb{N}}$.

Let $m \in \mathcal{M}^{1}\left(\Lambda^{\mathbb{N}}\right)$ be $\operatorname{Incr}(\mathbb{N})$-invariant, and let us prove that $m \operatorname{in} \operatorname{Inj}(\mathbb{N})$ invariant. So let $\sigma \in \operatorname{Inj}(\mathbb{N})$. We must show that

$$
\int_{\Lambda^{\mathbb{N}}} g d m=\int_{\Lambda^{\mathbb{N}}} g \circ \sigma^{*} d m
$$

for all $g \in C\left(\Lambda^{\mathbb{N}}\right)$. It suffices to prove (B.3) for $g$ in a dense subset $D$ of $C\left(\Lambda^{\mathbb{N}}\right)$. So we can assume that $g(x)$ has the form $g_{0}\left(x_{0}\right) \cdot \ldots \cdot g_{r}\left(x_{r}\right)$ for some $r \in \mathbb{N}$ and $g_{1}, \ldots, g_{r} \in C(\Lambda)$. Note that $g_{i}\left(x_{i}\right)=\left(g_{i} \circ P_{i}\right)(x)$ where $P_{i}: \Lambda^{\mathbb{N}} \rightarrow \Lambda$ is the projection on the $i$-th coordinate. Since $P_{i}=P_{0} \circ \mathrm{S}^{*}$ where $S^{*}$ is the shift, we can apply Lemma B.4 to obtain

$$
\int_{\Lambda^{\mathbb{N}}} g d m=\int_{\Lambda^{\mathbb{N}}} \widetilde{g_{1} \circ P_{1}} \cdots \widetilde{g_{r} \circ P_{1}} d m .
$$

Reasoning in the same way for the function $g \circ \sigma^{*}$, we finally get

$$
\int_{\Lambda^{\mathbb{N}}} g \circ \sigma^{*} d m=\int_{\Lambda^{\mathbb{N}}} \widetilde{g_{1} \circ P_{1}} \cdots \widetilde{g_{r} \circ P_{1}} d m=\int_{\Lambda^{\mathbb{N}}} g d m .
$$

Definition B.5. We say that $m \in \mathcal{M}^{1}\left(\Lambda^{\mathbb{N}}\right)$ is asymptotically exchangeable if the limit $m^{\prime}=\lim _{\theta \in \operatorname{Incr}(\mathbb{N})} \theta \cdot m$ exists in $\mathcal{M}^{1}\left(\Lambda^{\mathbb{N}}\right)$ and is an exchangeable measure.

Remark B.6. Note that if $m$ is asymptotically exchangeable, then:

$$
\begin{aligned}
m^{\prime} & :=\lim _{\substack{\min \theta \rightarrow \infty \\
\operatorname{Incr}(\mathbb{N})}} \theta \cdot m \\
& =\lim _{k \rightarrow \infty} \mathrm{S}^{k} \cdot m .
\end{aligned}
$$

However it is possible that $\lim _{k \rightarrow \infty} \mathrm{S}^{k} \cdot m$ exists and is exchangeable, and yet $m$ is not asymptotically exchangeable. As an example one may start with the Bernoulli probability measure $\mu$ on $2^{\mathbb{N}}$ with $\mu\left(\left\{x_{i}=0\right\}\right)=1 / 2$ and then 
consider the conditional probability $m(\cdot)=\mu(\cdot \mid A)$ where $A \subset 2^{\mathbb{N}}$ is the set of those sequences $x \in 2^{\mathbb{N}}$ satisfying $x_{(n+1)^{2}}=1-x_{n^{2}}$ for all $n$.

Remark B.7. If $m$ is asymptotically exchangeable and $m^{\prime}=\lim _{k \rightarrow \infty} \mathrm{S}^{k} \cdot m$, then for all $r \in \mathbb{N}$ and $g_{1}, \ldots, g_{r} \in C(\Lambda)$ we have

$$
\lim _{\substack{i_{1} \rightarrow+\infty \\\left(i_{1}, \ldots, i_{r}\right) \in \mathbb{N}(r)}} \int_{\Lambda^{\mathbb{N}}} g_{1}\left(x_{i_{1}}\right) \cdots g_{r}\left(x_{i_{r}}\right) d m=\int_{\Lambda^{\mathbb{N}}} g_{1}\left(x_{1}\right) \cdots g_{r}\left(x_{r}\right) d m^{\prime} .
$$

Theorem B.8. Given $m \in \mathcal{M}^{1}\left(\Lambda^{\mathbb{N}}\right)$ there is $\sigma \in \omega^{(\omega)}$ such that $\sigma \cdot m$ is asymptotically exchangeable.

Proof. Fix $m \in \mathcal{M}^{1}\left(\Lambda^{\mathbb{N}}\right)$. Given $r \in \omega$ consider the function $f: \omega^{(r)} \rightarrow$ $\mathcal{M}^{1}\left(\Lambda^{r}\right)$ sending $\iota$ to $\iota \cdot m \in \mathcal{M}^{1}\left(\Lambda^{r}\right)$. By Lemma A.1 there is an infinite set $J_{r} \subset \omega$ such that

$$
\lim _{\substack{\min (\iota) \rightarrow \infty \\ \iota \in J_{r}^{(r)}}} \iota \cdot m
$$

exists in $\mathcal{M}^{1}\left(\Lambda^{r}\right)$. By a diagonal argument we choose the same set $J=J_{r}$ for all $r$. Let $\sigma \in \operatorname{Incr}(\mathbb{N})$ be such that $\sigma(\mathbb{N})=J$. We claim that $\sigma \cdot m$ is asymptotically exchangeable. To this aim consider $m_{k}:=\mathrm{S}^{k} \cdot \sigma \cdot m \in$ $\mathcal{M}^{1}\left(\Lambda^{\mathbb{N}}\right)$. By compactness there is an accumulation point $m^{\prime} \in \mathcal{M}^{1}\left(\Lambda^{\mathbb{N}}\right)$ of $\left\{m_{k}\right\}_{k \in \mathbb{N}}$. We claim that

$$
\lim _{\substack{\min (\theta) \rightarrow J^{(\omega)} \\ \theta \in}} \theta \cdot \sigma \cdot m=m^{\prime}
$$

hence in particular $m_{k} \rightarrow m^{\prime}$ (taking $\theta=\mathrm{S}^{k}$ ). Note that the claim also implies that $m^{\prime}$ is exchangeable. Indeed, given an increasing function $\gamma: \mathbb{N} \rightarrow$ $\mathbb{N}$, to show $\gamma \cdot m^{\prime}=m^{\prime}$ it suffices to replace $\theta$ with $\theta \circ \gamma$ in equation (B.8). Since the subset of $C\left(\Lambda^{\mathbb{N}}\right)$ consising of the functions depending on finitely many coordinates is dense, it suffices to prove that for all $r \in \mathbb{N}$ and $\iota \in \mathbb{N}^{(r)}$ the limit

$$
\lim _{\substack{\min (\theta) \rightarrow \infty \\ \theta \in J(\omega)}} \iota \cdot \theta \cdot \sigma \cdot m
$$

exists in $\mathcal{M}^{1}\left(\Lambda^{r}\right)$ (the limit being necessarily $\iota \cdot m^{\prime}$ ). This is however just a special case of equation B.7.

We give below some representation results for exchangeable measures. First note that if $\Lambda$ is countable, a measure $m \in \mathcal{M}^{1}\left(\Lambda^{\mathbb{N}}\right)$ is determined by the values it takes on the sets of the form $\left\{x: x_{i_{1}}=a_{1}, \ldots, x_{i_{r}}=a_{r}\right\}$.

Lemma B.9. If $\Lambda$ is countable, a measure $m \in \mathcal{M}\left(\Lambda^{\mathbb{N}}\right)$ is exchangeable if and only if it admits a representation of the following form. There is a probability space $(\Omega, \mu)$ (which in fact can be taken to be $\left(\Lambda^{\mathbb{N}}, m\right)$ ) and a family $\left\{\psi_{a}\right\}_{a \in \Lambda}$ in $L^{\infty}(\Omega, \mu)$ such that for all $i_{1}<\ldots<i_{r}$ in $\mathbb{N}$ we have

$$
m\left(\left\{x: x_{i_{1}}=a_{1}, \ldots, x_{i_{r}}=a_{r}\right\}\right)=\int_{\Omega} \psi_{a_{1}} \cdot \ldots \cdot \psi_{a_{n}} d \mu .
$$


Proof. Since the right-hand side of the equation does not depend on $i_{1}, \ldots, i_{r}$ a measure $m \in \mathcal{M}^{1}\left(\Lambda^{\mathbb{N}}\right)$ admitting the above representation is clearly exchangeable. Conversely if $m$ is exchangeable it suffices to take $\psi_{a}=\widetilde{\chi_{a}}$ where $\chi_{a}$ is the characteristic function of the set $\left\{x: x_{0}=a\right\}$. We can in fact obtain the desired result by a repeated application of Equation (B.2) after observing that the characteristic function $\chi_{\left\{x: x_{i_{1}}=a_{1}, \ldots, x_{i_{r}}=a_{r}\right\}}$ is the product $\chi_{\left\{x: x_{i_{1}}=a_{1}\right\}} \cdot \ldots \cdot \chi_{\left\{x_{i_{r}}=a_{r}\right\}}$ and $\chi_{\left\{x: x_{i}=a\right\}}=\chi_{a} \circ\left(\mathrm{S}^{*}\right)^{i}$.

Corollary B.10. If $\Lambda$ is countable and $m \in \mathcal{M}^{1}\left(\Lambda^{\mathbb{N}}\right)$ is exchangeable, then $m\left(\left\{x \in \Lambda^{\mathbb{N}}: x_{0}=x_{1}\right\}\right) \neq 0$.

Proof. By $\left(\right.$ B.10) $m\left(\left\{x \in \Lambda^{\mathbb{N}}: x_{0}=x_{1}\right\}\right)=\sum_{a \in \Lambda} \int \psi_{a}^{2} d \mu \neq 0$.

Corollary B.11. If $p \in \mathbb{N}$ and $m \in \mathcal{M}^{1}\left(p^{\mathbb{N}}\right)$ is exchangeable, then $m(\{x \in$ $\left.\left.\Lambda^{\mathbb{N}}: x_{0}=x_{1}\right\}\right) \geq \frac{1}{p}$.

Proof. Write $m\left(\left\{x \in \Lambda^{\mathbb{N}}: x_{0}=x_{1}\right\}\right)=\sum_{a \in \Lambda} \int_{\Omega} \psi_{a}{ }^{2}$ and apply the CauchySchwarz inequality to the linear operator $\sum \int$ on $p \times \Omega$ to obtain

$$
\left(\sum_{a<p} \int_{\Omega} \psi_{a}^{2} d \mu\right) \cdot\left(\sum_{a<p} \int_{\Omega} 1 d \mu\right) \geq\left(\sum_{a<p} \int_{\Omega} \psi_{a} d \mu\right)^{2}
$$

which gives the desired result.

Thanks to a theorem of De Finetti, suitably extended in HS:55 there is an integral representation à la Choquet for the exchangeable measures on $\Lambda^{\mathbb{N}}$, where $\Lambda$ is a compact metric space. More precisely, in [HS:55] it is shown that the extremal points of the (compact) convex set of all exchangeable measures are given by the product measures $\sigma^{\mathbb{N}}$, with $\sigma \in \mathcal{M}^{1}(\Lambda)$. As a consequence, Choquet theorem C:69 provides an integral representation for any exchangeable measure $m$ on $\Lambda^{\mathbb{N}}$, i.e. there is a probability measure $\mu \in \mathcal{M}^{1}(\Lambda)$ such that

$$
m=\int_{\mathcal{M}^{1}(\Lambda)} \sigma^{\mathbb{N}} d \mu(\sigma) .
$$

When $\Lambda$ is finite, i.e. $\Lambda=p=\{0, \ldots, p-1\}$ for some $p \in \mathbb{N}$, we can identify $\mathcal{M}^{1}(\Lambda)$ with the symplex $\Sigma_{p}$ of all $\lambda \in[0,1]^{p}$ such that $\sum_{i=0}^{p-1} \lambda_{i}=1$. Given $\lambda \in \Sigma_{p}$, we denote by $B_{\lambda}$ the product measure on $p^{\mathbb{N}}$, namely the unique measure making all the events $\left\{x: x_{i}=a\right\}$ independent with measure $B_{\lambda}\left(\left\{x: x_{i}=a\right\}\right)=\lambda_{a}$. In this case, (B.12) becomes

$$
m=\int_{\Sigma_{p}} B_{\lambda} d \mu(\lambda)
$$

where $\mu$ is a probability measure on $\Sigma_{p}$.

We finish this excursus on exchangeable measures with the following result:

Proposition B.12. Let $m \in \mathcal{M}^{1}\left(\Lambda^{\mathbb{N}}\right)$ be exchangeable, then for all $f \in$ $L^{1}\left(\Lambda^{\mathbb{N}}\right)$ the following conditions are equivalent:

a) $f$ is $\mathfrak{S}_{c}(\mathbb{N})$-invariant;

b) $f$ is $\operatorname{Inj}(\mathbb{N})$-invariant; 
c) $f$ is shift-invariant.

Proof. Since $\mathfrak{S}_{c}(\mathbb{N}) \subset \operatorname{Inj}(\mathbb{N})$ and $s \in \operatorname{Inj}(\mathbb{N})$, the implications b) $\Rightarrow$ a) and b) $\Rightarrow$ c) are obvious.

In order to prove that a) $\Rightarrow \mathrm{b}$ ), we let $\mathcal{F}=\left\{\sigma \in \operatorname{Inj}(\mathbb{N}): f=f \circ \sigma^{*}\right\}$, which is a closed subset of $\operatorname{Inj}(\mathbb{N})$ containing $\mathfrak{S}_{c}(\mathbb{N})$. Then, it is enough to observe that $\mathfrak{S}_{c}(\mathbb{N})$ is a dense subset of $\operatorname{Inj}(\mathbb{N}) \subset \mathbb{N}^{\mathbb{N}}$, with respect to the product topology of $\mathbb{N}^{\mathbb{N}}$, so that $\mathcal{F}=\overline{\mathfrak{S}_{c}(\mathbb{N})}=\operatorname{Inj}(\mathbb{N})$.

Let us prove that $\mathrm{c}) \Rightarrow \mathrm{a})$. Let $\sigma \in \mathfrak{S}_{c}(\mathbb{N})$ and let $n$ be such that $\sigma(i)=i$ for all $i \geq n$. It follows that $\mathrm{S}^{* k} \circ \sigma^{*}=\mathrm{S}^{k}$, for all $k \geq n$. As a consequence, for $m$-almost every $x \in \Lambda^{\mathbb{N}}$ it holds

$$
f \circ \sigma^{*}(x)=f \circ \mathrm{S}^{* n} \circ \sigma^{*}(x)=f \circ \mathrm{S}^{* n}(x)=f(x),
$$

where the first equality holds since the measure $m$ is $\mathfrak{S}_{c}(\mathbb{N})$-invariant.

Notice that from Proposition B.12 it follows that $\tilde{f}$ is $\operatorname{Inj}(\mathbb{N})$-invariant for all $f \in L^{1}\left(\Lambda^{\mathbb{N}}\right)$. In particular, for an exchangeable measure, the $\sigma$-algebra of the shift-invariant sets coincides with the (a priori smaller) $\sigma$-algebra of the $\operatorname{Inj}(\mathbb{N})$-invariant sets.

\section{REFERENCES}

[AFP:00] L. Ambrosio, N. Fusco, D. Pallara, Functions of bounded variation and free discontinuity problems, Oxford Mathematical monographs (2000).

[B:79] B. Bollobás, Graph Theory: An Introductory Course, Springer-Verlag, New York (1979).

[C:74] S. D. Chatterji, A subsequence principle in probability theory, Bull. of the AMS 80 (1974), 495-497.

[C:69] G. Choquet, Lectures on Analysis I, Benjamin, Reading, Mass. (1969).

[DF:74] B. De Finetti, Theory of Probability, Wiley, London (1974).

[PR:05] C. A. Di Prisco, Mathematics versus metamathematics in Ramsey theory of the real numbers, Proceedings of the 12th International Congress, King's College Publications (2005), 171-187.

[EH:64] P. Erdős, A. Hajnal, Some remarks on set theory. IX: Combinatorial problems in measure theory and set theory. Mich. Math. J. 11 (1964), 107-127.

[FS:76] T. Figiel, L. Sucheston, An application of Ramsey sets in analysis, Advances in Math. 20 (1976), 103-105.

[FT:85] D. H. Fremlin, M. Talagrand, Subgraphs of random graphs, Trans. Amer. Math. Soc. 291 (1985), 551-582.

[GP:73] F. Galvin, K. Prikry, Borel sets and Ramsey's theorem, Journal of Symbolic Logic 38 (1973), 193-198.

[G:99] G. Grimmett, Percolation, II ed., Springer-Verlag, Berlin (1999).

[HS:55] E. Hewitt and J. Savage, Symmetric measures on cartesian products, Trans. of the AMS 80 (1955), 470-501.

[K:05] O. Kallenberg, Probabilistic Symmetries and Invariance Principles, SpringerVerlag, New York (2005).

[K:95] A. S. Kechris, Classical descriptive set theory. Graduate Texts in Mathematics, 156. Springer-Verlag, New York, 1995. xviii+402 pp.

[K:78] J. F. C. Kingman, Uses of exchangeability, Annals of Prob. 6 (1978), 183-197.

[P:82] W. Parry, Topics in Ergodic Theory, Cambridge University Press, Cambridge (1982).

[R:28] F. P. Ramsey, On a problem of formal logic, Proc. London Math. Soc., Ser. 2, 30 (1928), 338-384.

[R:82] C. R. Rao, Diversity and dissimilarity coefficients: a unified approach, Theoretical Population Biology, 21 (1982), 24-43. 
Alessandro Berarducci, Dipartimento di Matematica, Università di Pisa, Largo B. Pontecorvo 5, 56127 Pisa, Italy, email: berardu@dm.unipi.it

Pietro Majer, Dipartimento di Matematica, Università di Pisa, Largo B. PonteCORVo 5, 56127 PisA, ItAly, EMAIL: majer@dm.unipi.it

Matteo Novaga, Dipartimento di Matematica, Università di Padova, Via Trieste 63, 35121 PAdova, ItAly, EMAIL: novaga@math.unipd.it 